TRANSACTIONS OF THE

AMERICAN MATHEMATICAL SOCIETY

Volume 357, Number 5, Pages 1753-1778

S 0002-9947(04)03726-2

Article electronically published on December 22, 2004

\title{
SOLVING THE KORTEWEG-DE VRIES EQUATION BY ITS BILINEAR FORM: WRONSKIAN SOLUTIONS
}

\author{
WEN-XIU MA AND YUNCHENG YOU
}

\begin{abstract}
A broad set of sufficient conditions consisting of systems of linear partial differential equations is presented which guarantees that the Wronskian determinant solves the Korteweg-de Vries equation in the bilinear form. A systematical analysis is made for solving the resultant linear systems of second-order and third-order partial differential equations, along with solution formulas for their representative systems. The key technique is to apply variation of parameters in solving the involved non-homogeneous partial differential equations. The obtained solution formulas provide us with a comprehensive approach to construct the existing solutions and many new solutions including rational solutions, solitons, positons, negatons, breathers, complexitons and interaction solutions of the Korteweg-de Vries equation.
\end{abstract}

\section{INTRODUCTION}

Among integrable equations is the celebrated Korteweg-de Vries (KdV) equation, which serves as a model equation governing weakly nonlinear long waves whose phase speed attains a simple maximum for waves of infinite length [DJ]. It motivates us to explore beauty hidden in nonlinear differential (and difference) equations. The remarkable and exceptional discovery of the inverse scattering transform ASe is one of important developments in the field of applied mathematics, which comes from the study of the KdV equation. There are various algebraic and geometric characteristics that the KdV equation possesses, for example, infinitely many symmetries and infinitely many conserved densities [MGK], the Lax representation $[\mathrm{L}$, bi-Hamiltonian structure [Mag, loop group [SW], and the Darboux-Bäcklund transformation [MS]. More significantly, many physically important solutions to the $\mathrm{KdV}$ equation can be presented explicitly through a simple, specific form, called the Hirota bilinear form. Such exact solutions contain solitons $[\mathrm{H}, \mathrm{S}$, rational solutions ASa, positons [APP, Mat, [K], negatons [RSK, breathers [J] and complexitons [M].

Let us consider the KdV equation in its standard form

$$
u_{t}-6 u u_{x}+u_{x x x}=0 .
$$

Hirota $[\mathrm{H}]$ introduced the transformation (here and in the rest of the paper, we use the notation $\ln f$ for simplicity, which does not imply that $f>0$ )

$$
u=-2 \partial_{x}^{2} \ln f=-2(\ln f)_{x x}=-\frac{2\left(f f_{x x}-f_{x}^{2}\right)}{f^{2}},
$$

Received by the editors June 2, 2003.

2000 Mathematics Subject Classification. Primary 35Q53, 37K10; Secondary 35Q51, 37K40.

Key words and phrases. Integrable equation, soliton theory.

(c)2004 American Mathematical Society $1753^{\text {Reverts to public domain } 28 \text { years from publication }}$ 
and transformed the $\mathrm{KdV}$ equation (1.1) into the bilinear form

$$
\left(D_{x} D_{t}+D_{x}^{4}\right) f \cdot f=f_{x t} f-f_{t} f_{x}+f_{x x x x} f-4 f_{x x x} f_{x}+3 f_{x x}^{2}=0,
$$

which is called the bilinear $\mathrm{KdV}$ equation. Here $D_{x}$ and $D_{t}$ are Hirota's bilinear operators $[\mathrm{BC}]$, defined by

$$
f(x+h, t+k) g(x-h, t-k)=\sum_{i, j=0}^{\infty} \frac{1}{i ! j !}\left(D_{x}^{i} D_{t}^{j} f \cdot g\right) h^{i} k^{j},
$$

which are also closely related to the vertex operators from elementary particle theory. Strictly speaking, we have

$$
u_{t}-6 u u_{x}+u_{x x x}=-\left[\frac{2\left(D_{x} D_{t}+D_{x}^{4}\right) f \cdot f}{f^{2}}\right]_{x} .
$$

Therefore, if $f$ solves the bilinear $\mathrm{KdV}$ equation (1.2), then $u=-2 \partial_{x}^{2} \ln f$ solves the original $\mathrm{KdV}$ equation (1.1). The bilinear form (1.2) looks a little bit more complicated than the KdV equation (1.1) itself, but its bilinear property, the nearest neighbor of the linear property, brings us great convenience in constructing explicit exact solutions. Such a bilinear form exists not only for the KdV equation, but also for many other physically important soliton equations, such as the AblowitzKaup-Newell-Segur equations [AKNS], the Kadomtsev-Petviashvili equation [KP], and the Benney-Roskes equation [BR] (or the Davey-Stewartson equation [DS]).

The Wronskian technique is a powerful tool to construct exact solutions to bilinear differential (and difference) equations. To use this technique, we adopt the compact Freeman and Nimmo's notation [FN]:

$$
\begin{aligned}
(\widehat{N-1}) & =(\widehat{N-1} ; \Phi)=W\left(\phi_{1}, \phi_{2}, \cdots, \phi_{N}\right) \\
& =\left|\begin{array}{cccc}
\phi_{1}^{(0)} & \phi_{1}^{(1)} & \ldots & \phi_{1}^{(N-1)} \\
\phi_{2}^{(0)} & \phi_{2}^{(1)} & \cdots & \phi_{2}^{(N-1)} \\
\vdots & \vdots & \ddots & \vdots \\
\phi_{N}^{(0)} & \phi_{N}^{(1)} & \cdots & \phi_{N}^{(N-1)}
\end{array}\right|, N \geq 1,
\end{aligned}
$$

where

$$
\phi_{i}^{(0)}=\phi_{i}, \phi_{i}^{(j)}=\frac{\partial^{j}}{\partial x^{j}} \phi_{i}, j \geq 1,1 \leq i \leq N .
$$

Satsuma, Freeman and Nimmo $[\mathrm{S}[\mathrm{FN}]$ proved that multi-soliton solutions to the $\mathrm{KdV}$ equation can be expressed through the above Wronskian determinant, and afterwards, Matveev Mat] generalized the Wronskian determinant which allows us to present another important class of exact solutions, called positons, to the $\mathrm{KdV}$ equation. In using the Wronskian method to solve the KdV equation, one usually starts from

$$
-\phi_{i, x x}=\lambda_{i} \phi_{i}, \phi_{i, t}=-4 \phi_{i, x x x}, 1 \leq i \leq N,
$$

where $\lambda_{i}$ are arbitrary real constants, to guarantee that the Wronskian determinant (1.3) solves the bilinear $\mathrm{KdV}$ equation (1.2) and thus generates a solution

$$
u=-2 \partial_{x}^{2} \ln W\left(\phi_{1}, \phi_{2}, \cdots, \phi_{N}\right)
$$

to the KdV equation (1.1). The solution determined by a Wronskian determinant is called a Wronskian solution. For example, $f=W\left(\phi_{1}, \phi_{2}, \cdots, \phi_{n}\right)$ and $u=$ $-2 \partial_{x}^{2} \ln W\left(\phi_{1}, \phi_{2}, \cdots, \phi_{N}\right)$ are Wronskian solutions to the bilinear KdV equation 
(1.2) and the KdV equation (1.1), respectively, provided that $\phi_{1}, \phi_{2}, \cdots, \phi_{n}$ satisfy (1.5). Sirianunpiboon et al. [SHR] extended the conditions (1.5) to the following:

$$
-\phi_{i, x x}=\sum_{j=1}^{i} \lambda_{i j} \phi_{j}, \phi_{i, t}=-4 \phi_{i, x x x}, 1 \leq i \leq N,
$$

where $\lambda_{i j}$ are arbitrary real constants, in order that the Wronskian determinant can generate rational function solutions and their interaction solutions with multisolitons. The first group of the above conditions (1.7) is a triangular system of ordinary differential equations. Thus, solving the system of differential equations (1.7) is exactly the same for scalar differential equations.

In this paper, we would like to construct explicit exact solutions to the KdV equation (1.1) by its bilinear form and to illustrate the entire process of construction with a broader set of sufficient conditions on the Wronskian solutions:

$$
-\phi_{i, x x}=\sum_{j=1}^{N} \lambda_{i j} \phi_{j}, \phi_{i, t}=-4 \phi_{i, x x x}+\xi \phi_{i}, 1 \leq i \leq N,
$$

where $\lambda_{i j}$ and $\xi$ are arbitrary real constants. Here the first group of conditions is a coupled system of ordinary differential equations of second-order, and thus needs a more general consideration for solving the involved differential equations. Note that the coefficient matrix $\Lambda=\left(\lambda_{i j}\right)$ in (1.7) has only real eigenvalues, but the coefficient matrix $\Lambda=\left(\lambda_{i j}\right)$ in (1.8) in general has complex eigenvalues. This is a significant difference between two sets of conditions, defined by (1.7) and (1.8). The substantial extension in (1.8) allows us to get a much broader class of explicit exact solutions to the KdV equation (1.1). In particular, new exact solutions called complexitons can be generated from the set of conditions (1.8), but not (1.7).

Now the whole problem of constructing the Wronskian solutions to the KdV equation (1.1) reduces to solve the coupled system of partial differential equations (1.8), which turns out to be an interesting mathematical problem itself. We shall analyze solution structures on the system (1.8) in detail, to obtain solution formulas for all cases of the system (1.8). The resultant fundamental solution formulas provide a direct and comprehensive approach to construct diverse exact solutions to the KdV equation (1.1), such as rational solutions, solitons, positons, negatons, breathers, complexitons, and more generally their interaction solutions.

\section{SUfFICIENT CONDITIONS ON WRONSKIAN SOLUTIONS}

We begin with stating a broad set of sufficient conditions which make the Wronskian determinant a solution to the bilinear KdV equation (1.2).

Theorem 2.1. Assume that a group of functions $\phi_{i}=\phi_{i}(x, t), 1 \leq i \leq N$, satisfies the two sets of conditions

$$
\begin{gathered}
-\phi_{i, x x}=\sum_{j=1}^{N} \lambda_{i j}(t) \phi_{j}, 1 \leq i \leq N, \\
\phi_{i, t}=-4 \phi_{i, x x x}+\xi(t) \phi_{i}, 1 \leq i \leq N,
\end{gathered}
$$

simultaneously, where $\lambda_{i j}(t)$ are arbitrary differentiable real functions of $t$ and $\xi(t)$ is an arbitrary continuous real function of $t$. Then $f=(\widehat{N-1})$ defined by (1.3) solves the bilinear KdV equation (1.2). 
The conditions (2.1) and (2.2) are a generalization to (1.7) presented by Sirianunpiboon et al. $\left[\mathrm{SHR}\right.$. Actually, if $\xi=0$ and the coefficient matrix $\Lambda=\left(\lambda_{i j}\right)$ of (2.1) is lower-triangular and independent of time $t$, then the result of the above theorem boils down to the result in $[\mathrm{SHR}$. The proof of the theorem needs some basic equalities, and the following notation is helpful in our deduction and analysis:

$$
\begin{aligned}
& \left(N \widehat{-j-1}, i_{1}, \cdots, i_{j}\right)=\left|\Phi^{(0)}, \Phi^{(1)}, \cdots, \Phi^{(N-j-1)}, \Phi^{\left(i_{1}\right)}, \cdots, \Phi^{\left(i_{j}\right)}\right| \\
& =\operatorname{det}\left(\Phi^{(0)}, \Phi^{(1)}, \cdots, \Phi^{(N-j-1)}, \Phi^{\left(i_{1}\right)}, \cdots, \Phi^{\left(i_{j}\right)}\right), 1 \leq j \leq N-1,
\end{aligned}
$$

where $i_{1}, \cdots, i_{j}$ are non-negative integers, and the vectors of functions $\Phi^{(j)}$ are defined by

$$
\Phi^{(j)}=\left(\phi_{1}^{(j)}, \phi_{2}^{(j)}, \cdots, \phi_{N}^{(j)}\right)^{T}, 0 \leq j \leq N-1 .
$$

We also use the assumption for convenience that if $i<0$, the column vector $\Phi^{(i)}$ will disappear in the determinant $\operatorname{det}\left(\cdots, \Phi^{(i)}, \cdots\right)$.

Lemma 2.2. Under the conditions (2.1), the following equalities hold:

$$
\begin{aligned}
& \sum_{i=1}^{N} \lambda_{i i}(t)(\widehat{N-1})=(\widehat{N-3}, N-1, N)-(\widehat{N-2}, N+1), \\
& \frac{\partial^{2}(\widehat{N-1})}{\partial x^{2}}=-\sum_{i=1}^{N} \lambda_{i i}(t)(\widehat{N-1})+2(\widehat{N-3}, N-1, N), \\
& \sum_{i=1}^{N} \lambda_{i i}(t)(\widehat{N-3}, N-1, N)=(\widehat{N-5}, N-3, N-2, N-1, N) \\
& \quad+(\widehat{N-3}, N, N+1)-(\widehat{N-3}, N-1, N+2),
\end{aligned}
$$

and

$$
\begin{aligned}
& \left(\sum_{i=1}^{N} \lambda_{i i}(t)\right)^{2}(\widehat{N-1})=(\widehat{N-5}, N-3, N-2, N-1, N) \\
& \quad-(\widehat{N-4}, N-2, N-1, N+1)-(\widehat{N-3}, N-1, N+2) \\
& \quad+2(\widehat{N-3}, N, N+1)+(\widehat{N-2}, N+3) .
\end{aligned}
$$

Proof. Note that we have

$$
\sum_{k=1}^{N}|A|_{k l}=\sum_{k=1}^{N}|A|^{k l}=\sum_{i, j=1}^{N} A_{i j} \frac{\partial^{l} a_{i j}}{\partial x^{l}},
$$

where $A=\left(a_{i j}\right)_{N \times N}$, and $|A|_{k l},|A|^{k l}$ and $A_{i j}$ denote the determinant resulting from $|A|$ with its $k$ th row differentiated $l$ times with respect to $x$, the determinant resulting from $|A|$ with its $k$ th column differentiated $l$ times with respect to $x$, and the co-factor of $a_{i j}$, respectively. Choose $|A|$ as $(\widehat{N-1})$ and $(\widehat{N-3}, N-1, N)$, and use the above equality with $l=2$ and the conditions (2.1). Then we obtain the required equalities (2.5) and (2.7) immediately. A combination of the equality (2.5) and the equality

$$
\frac{\partial^{2}(\widehat{N-1})}{\partial x^{2}}=(\widehat{N-3}, N-1, N)+(\widehat{N-2}, N+1)
$$

leads to the equality (2.6). 
We now differentiate (2.5) twice with respect to $x$ and utilize (2.6) and (2.7). Then a further computation yields the equality (2.8). This completes the proof of the lemma.

Proof of Theorem 2.1. By using the conditions (2.2), we obtain that

$$
\begin{aligned}
f_{t}= & N \xi f-4[(\widehat{N-4}, N-2, N-1, N) \\
& -(\widehat{N-3}, N-1, N+1)+(\widehat{N-2}, N+2)], \\
f_{x t}= & N \xi f_{x}-4[(\widehat{N-5}, N-3, N-2, N-1, N) \\
& -(\widehat{N-3}, N, N+1)+(\widehat{N-2}, N+3)] .
\end{aligned}
$$

Therefore, we can further deduce that

$$
\begin{aligned}
\Delta:= & D_{x}\left(D_{t}+D_{x}^{3}\right) f \cdot f=f_{x t} f-f_{t} f_{x}+f_{x x x x} f-4 f_{x x x} f_{x}+3 f_{x x}^{2} \\
= & {[-3(\widehat{N-5}, N-3, N-2, N-1, N)+6(\widehat{N-3}, N, N+1)-3(\widehat{N-2}, N+3)} \\
& +3(\widehat{N-4}, N-2, N-1, N+1)+3(\widehat{N-3}, N-1, N+2)](\widehat{N-1}) \\
& -12(\widehat{N-3}, N-1, N+1)(\widehat{N-2}, N) \\
& +3[(\widehat{N-3}, N-1, N)+(\widehat{N-2}, N+1)]^{2} .
\end{aligned}
$$

Now using the equalities (2.5) and (2.8) in Lemma 2.2 and the Laplace expansion of determinants about the last $N$ rows, we have

$$
\begin{aligned}
\Delta & =-12(\widehat{N-3}, N-1, N+1)(\widehat{N-2}, N) \\
& +12(\widehat{N-3}, N, N+1)(\widehat{N-1})+12(\widehat{N-3}, N-1, N)(\widehat{N-2}, N+1) \\
= & 6\left|\begin{array}{cccccc}
\widehat{N-3} & 0 & N-2 & N-1 & N & N+1 \\
0 & \widehat{N-3} & N-2 & N-1 & N & N+1
\end{array}\right|=0 .
\end{aligned}
$$

This shows that $f=(\widehat{N-1})$ solves (1.2). Note that the entries in the last abbreviated $2 N \times 2 N$ determinant above are derivatives of $\Phi$, e.g., $N-1$ denotes $\Phi^{(N-1)}$. The proof is finished.

Theorem 2.1 tells us that if a group of functions $\phi_{i}(x, t), 1 \leq i \leq N$, satisfies the conditions (2.1) and (2.2), then we can get a solution $f=W\left(\phi_{1}, \phi_{2}, \cdots, \phi_{N}\right)$ to the bilinear KdV equation (1.2). The conditions (2.2) and (2.1) are two linear systems of second-order and third-order partial differential equations. We are going to solve these linear systems explicitly. It is rather difficult to deal with the case where the coefficient matrix $\Lambda$ of (2.1) has complex eigenvalues. However, when $-\Lambda$ is a positive definite constant matrix, the situation is easy and the solution formula of (2.1) and (2.2) can be expressed as

$$
\Phi=\mathrm{e}^{\int_{0}^{t} \xi\left(t^{\prime}\right) d t^{\prime}} \mathrm{e}^{\sqrt{-\Lambda} x+4 \Lambda \sqrt{-\Lambda} t} \Phi_{0}
$$

where $\sqrt{-\Lambda}$ is the square root of $-\Lambda$ and $\Phi_{0}$ is an arbitrary initial vector. The corresponding Wronskian solutions to the KdV equation (1.1),

$$
u=-2 \partial_{x}^{2} \ln f=-2 \partial_{x}^{2} \ln (\widehat{N-1} ; \Phi),
$$

contain soliton and negaton solutions.

Before we proceed to solve (2.1) and (2.2), let us observe the Wronskian determinants and solutions more carefully. 
Oservation I. From the compatibility conditions $\phi_{i, t x x}=\phi_{i, x x t}, 1 \leq i \leq N$, of the conditions (2.1) and (2.2), we have the equality

$$
\sum_{j=1}^{N} \lambda_{i j, t} \phi_{j}=0,1 \leq i \leq N
$$

and thus it is easy to see that the Wronskian determinant $W\left(\phi_{1}, \phi_{2}, \cdots, \phi_{N}\right)$ becomes zero if there is at least one entry $\lambda_{i j}$ satisfying $\lambda_{i j, t} \neq 0$.

Oservation II. If we make use of the transformation

$$
\tilde{\phi}_{i}=\mathrm{e}^{-\int_{0}^{t} \xi(s) d s} \phi_{i}, 1 \leq i \leq N,
$$

the differential equations in (2.2) can be put into

$$
\tilde{\phi}_{i, t}=-4 \tilde{\phi}_{i, x x x}, 1 \leq i \leq N
$$

but the differential equations (2.1) do not change. Obviously, the resultant Wronskian solutions to the KdV equation are the same:

$$
u=-2 \partial_{x}^{2} \ln W\left(\phi_{1}, \phi_{2}, \cdots, \phi_{N}\right)=\tilde{u}=-2 \partial_{x}^{2} \ln W\left(\tilde{\phi}_{1}, \tilde{\phi}_{2}, \cdots, \tilde{\phi}_{N}\right) .
$$

But the Wronskian determinants $W\left(\phi_{1}, \phi_{2}, \cdots, \phi_{N}\right)$ and $W\left(\tilde{\phi}_{1}, \tilde{\phi}_{2}, \cdots, \tilde{\phi}_{N}\right)$ are different, and thus they gives rise to different solutions to the bilinear KdV equation (1.2).

Oservation III. If the coefficient matrix $\Lambda=\left(\lambda_{i j}\right)$ is similar to another matrix $M=\left(\mu_{i j}\right)$ under an invertible constant matrix $P$, let us say $\Lambda=P^{-1} M P$, then $\tilde{\Phi}=P \Phi$ solves

$$
\tilde{\Phi}_{x x}=M \tilde{\Phi}, \tilde{\Phi}_{t}=-4 \tilde{\Phi}_{x x x}+\xi \tilde{\Phi}
$$

and the resultant Wronskian solutions to the $\mathrm{KdV}$ equation are also the same:

$$
\begin{aligned}
u(\Lambda) & =-2 \partial_{x}^{2} \ln \left|\Phi^{(0)}, \Phi^{(1)}, \cdots, \Phi^{(N-1)}\right| \\
& =-2 \partial_{x}^{2} \ln \left|P \Phi^{(0)}, P \Phi^{(1)}, \cdots, P \Phi^{(N-1)}\right|=u(M) .
\end{aligned}
$$

It follows from Observations II and III that different Wronskian determinants may lead to the same solution to the KdV equation. Therefore, based on Observation I, in order to construct Wronskian solutions to the KdV equation by its bilinear form, we only need to consider the reduced case of (2.2) and (2.1) under $\xi=0$ and $d \Lambda / d t=0$, i.e., the following conditions:

$$
-\phi_{i, x x}=\sum_{j=1}^{N} \lambda_{i j} \phi_{j}, \phi_{i, t}=-4 \phi_{i, x x x}, 1 \leq i \leq N,
$$


where $\lambda_{i j}$ are arbitrary real constants. On the other hand, the Jordan form of a real matrix has the following two types of blocks:

$$
\begin{aligned}
& {\left[\begin{array}{cccc}
\lambda_{i} & & & 0 \\
1 & \lambda_{i} & & \\
& \ddots & \ddots & \\
0 & & 1 & \lambda_{i}
\end{array}\right]_{k_{i} \times k_{i}},} \\
& {\left[\begin{array}{cccc}
A_{i} & & & 0 \\
I_{2} & A_{i} & & \\
& \ddots & \ddots & \\
0 & & I_{2} & A_{i}
\end{array}\right]_{l_{i} \times l_{i}}, A_{i}=\left[\begin{array}{cc}
\alpha_{i} & -\beta_{i} \\
\beta_{i} & \alpha_{i}
\end{array}\right], I_{2}=\left[\begin{array}{ll}
1 & 0 \\
0 & 1
\end{array}\right],}
\end{aligned}
$$

where $\lambda_{i}, \alpha_{i}$ and $\beta_{i}>0$ are all real constants. The first type of blocks has the real eigenvalue $\lambda_{i}$ with algebraic multiplicity $k_{i}$, and the second type of blocks has the complex eigenvalues $\lambda_{i}^{ \pm}=\alpha_{i} \pm \beta_{i} \sqrt{-1}$ with algebraic multiplicity $l_{i}$. Note that an eigenvalue of the coefficient matrix $\Lambda=\left(\lambda_{i j}\right)$ is also an eigenvalue of the Schrödinger operator $-\frac{\partial^{2}}{\partial x^{2}}+u$ with zero potential. We will present solution formulas for the system of differential equations defined by (2.16), according to the situations of eigenvalues of the coefficient matrix. Now, based on Observation III, all we need to do is to solve a group of subsystems of the sufficient conditions on the Wronskian solutions, whose coefficient matrices are of the forms (2.17) and (2.18).

Let us summarize the above analysis as the following theorem.

Theorem 2.3. For the KdV equation (1.1), all Wronskian solutions generated from the conditions (2.1) and (2.2) are among the Wronskian solutions associated with the special cases of the conditions (2.16) whose coefficient matrices $\Lambda=\left(\lambda_{i j}\right)$ are of the Jordan form consisting of two types of Jordan blocks in (2.17) and (2.18).

In the next section, we shall consider how to solve the two types of subsystems associated with the Jordan blocks in (2.17) and (2.18), and present solution formulas for their representative systems generating Wronskian solutions to the KdV equation (1.1).

\section{Solution FORMulas FOR THE REPRESENTATIVE SYSTEMS}

In this section, we would like to consider the construction of solutions to the associated system of differential equations defined by (2.16). Based on the form of two types of Jordan blocks in (2.17) and (2.18), a basic idea to solve the system (2.16) is to use a recursion process. That is to solve (2.16) from $\phi_{1}$ to $\phi_{N}$, one by one when the coefficient matrix $\Lambda=\left(\lambda_{i j}\right)$ has real eigenvalues or pair by pair when the coefficient matrix $\Lambda=\left(\lambda_{i j}\right)$ has complex eigenvalues. Therefore, the entire problem is divided into two subproblems - to solve the following two representative systems of non-homogeneous differential equations:

$$
-\phi_{x x}=\lambda \phi+f, \phi_{t}=-4 \phi_{x x x}
$$


and

$$
\left\{\begin{array}{c}
-\phi_{1, x x}=\alpha \phi_{1}-\beta \phi_{2}+f_{1}, \\
-\phi_{2, x x}=\beta \phi_{1}+\alpha \phi_{2}+f_{2}, \\
\phi_{i, t}=-4 \phi_{i, x x x}, i=1,2,
\end{array}\right.
$$

where $\lambda, \alpha$ and $\beta>0$ are real constants, and $f=f(x, t), f_{1}=f_{1}(x, t)$ and $f_{2}=$ $f_{2}(x, t)$ are three given functions satisfying the compatibility condition $g_{t}=-4 g_{x x x}$. The whole system (2.16) whose coefficient matrix is of Jordan form decouples into a group of systems determined by (3.1) and (3.2).

3.1. The case of real eigenvalues. First, let us consider the first representative system (3.1). In terms of the eigenvalue $\lambda$, we will establish solution formulas for three situations of the representative system (3.1).

Zero eigenvalue: The representative system (3.1) in this case is

$$
\phi_{x x}=f, \phi_{t}=-4 \phi_{x x x},
$$

where $f=f(x, t)$ is a given function satisfying the compatibility condition $f_{t}=$ $-4 f_{x x x}$. Immediately from the first equation in (3.3), we can get

$$
\phi=\int_{0}^{x} \int_{0}^{x^{\prime}} f\left(x^{\prime \prime}, t\right) d x^{\prime \prime} d x^{\prime}+c(t) x+d(t) .
$$

Note that $f_{t}=-4 f_{x x x}$, and thus the second equation of (3.3) equivalently requires

$$
c_{t}=-\left.4 f_{x x}\right|_{x=0}, d_{t}=-\left.4 f_{x}\right|_{x=0} .
$$

Then, we obtain

$$
c(t)=-4 \int_{0}^{t} f_{x x}\left(0, t^{\prime}\right) d t^{\prime}+c_{0}, d(t)=-4 \int_{0}^{t} f_{x}\left(0, t^{\prime}\right) d t^{\prime}+d_{0},
$$

where $c_{0}$ and $d_{0}$ are arbitrary real constants. Summing up, we have the following theorem.

Theorem 3.1. Let $f=f(x, t)$ be a given function satisfying the compatibility condition $f_{t}=-4 f_{x x x}$. Then the system of differential equations (3.3) has the general solution given by (3.4) and (3.5).

Negative eigenvalue: In this case, the representative system (3.1) is

$$
\phi_{x x}=\alpha \phi+f, \phi_{t}=-4 \phi_{x x x}, \alpha>0,
$$

where $\alpha$ is a constant and $f$ is a given function satisfying the compatibility condition $f_{t}=-4 f_{x x x}$. From two basic solutions $\mathrm{e}^{\sqrt{\alpha} x}$ and $\mathrm{e}^{-\sqrt{\alpha} x}$ of $\phi_{x x}=\alpha \phi$, we get a general solution of the first differential equation of (3.6):

$$
\begin{aligned}
\phi= & {\left[\frac{1}{2 \sqrt{\alpha}} \int_{0}^{x} f\left(x^{\prime}, t\right) \mathrm{e}^{-\sqrt{\alpha} x^{\prime}} d x^{\prime}+c(t)\right] \mathrm{e}^{\sqrt{\alpha} x} } \\
& -\left[\frac{1}{2 \sqrt{\alpha}} \int_{0}^{x} f\left(x^{\prime}, t\right) \mathrm{e}^{\sqrt{\alpha} x^{\prime}} d x^{\prime}+d(t)\right] \mathrm{e}^{-\sqrt{\alpha} x},
\end{aligned}
$$

by variation of parameters. Then noting that $f_{t}=-4 f_{x x x}$, by a direct but lengthy computation, we find that the second differential equation of (3.6) equivalently 
requires

$$
\begin{aligned}
& c_{t}=-4 \alpha \sqrt{\alpha} c-\left.2\left(\frac{1}{\sqrt{\alpha}} f_{x x}+f_{x}+\sqrt{\alpha} f\right)\right|_{x=0}, \\
& d_{t}=4 \alpha \sqrt{\alpha} d+\left.2\left(-\frac{1}{\sqrt{\alpha}} f_{x x}+f_{x}-\sqrt{\alpha} f\right)\right|_{x=0} .
\end{aligned}
$$

These are two linear systems for $c$ and $d$, respectively. Hence, we can immediately obtain the solution formulas for $c$ and $d$ :

$$
\begin{aligned}
& \left.c(t)=\mathrm{e}^{-4 \alpha \sqrt{\alpha} t}\left[c_{0}-2 \int_{0}^{t} \mathrm{e}^{4 \alpha \sqrt{\alpha} t^{\prime}}\left(\frac{1}{\sqrt{\alpha}} f_{x x}+f_{x}+\sqrt{\alpha} f\right)\left(0, t^{\prime}\right)\right) d t^{\prime}\right] \\
& \left.d(t)=\mathrm{e}^{4 \alpha \sqrt{\alpha} t}\left[d_{0}+2 \int_{0}^{t} \mathrm{e}^{-4 \alpha \sqrt{\alpha} t^{\prime}}\left(-\frac{1}{\sqrt{\alpha}} f_{x x}+f_{x}-\sqrt{\alpha} f\right)\left(0, t^{\prime}\right)\right) d t^{\prime}\right]
\end{aligned}
$$

where $c_{0}$ and $d_{0}$ are arbitrary real constants. We conclude this result as follows.

Theorem 3.2. Let $f=f(x, t)$ be a given function satisfying the compatibility condition $f_{t}=-4 f_{x x x}$. Then the system of differential equations (3.6) has the general solution given by (3.7), (3.8) and (3.9).

Positive eigenvalue: The representative system (3.1) in this case is

$$
\begin{aligned}
& \phi_{x x}=-\alpha \phi+f, \alpha>0, \\
& \phi_{t}=-4 \phi_{x x x},
\end{aligned}
$$

where $\alpha$ is a constant and $f=f(x, t)$ is a given function satisfying the compatibility condition $f_{t}=-4 f_{x x x}$. To solve the differential equation (3.10), we can start from two basic solutions $\sin \sqrt{\alpha} x$ and $\cos \sqrt{\alpha} x$ of the corresponding homogeneous equation of (3.10) , and then use the method of variation of parameters. A general solution is listed in the following lemma for reference.

Lemma 3.3. For any given function $g=g(x)$ and any positive constant $\alpha$, the second-order ordinary differential equation $\psi_{x x}=-\alpha \psi+g$ has the general solution

$$
\psi=\int_{0}^{x} \frac{\sin \sqrt{\alpha}\left(x-x^{\prime}\right)}{\sqrt{\alpha}} g\left(x^{\prime}\right) d x^{\prime}+c \cos \sqrt{\alpha} x+d \sin \sqrt{\alpha} x,
$$

where $c$ and $d$ are arbitrary real constants.

Now to solve the partial differential equation (3.11), we start with the solution of (3.10) given by Lemma 3.3 and use the method of variation of parameters again. Therefore, the solution of (3.11) is assumed to be

$$
\phi=\int_{0}^{x} \frac{\sin \sqrt{\alpha}\left(x-x^{\prime}\right)}{\sqrt{\alpha}} f\left(x^{\prime}, t\right) d x^{\prime}+c(t) \cos \sqrt{\alpha} x+d(t) \sin \sqrt{\alpha} x .
$$

Directly we can compute that

$$
\begin{aligned}
\phi_{x x x}= & f_{x}-\int_{0}^{x} f\left(x^{\prime}, t\right)\left(\alpha \cos \sqrt{\alpha}\left(x-x^{\prime}\right)\right) d x^{\prime} \\
& +c(t) \alpha \sqrt{\alpha} \sin \sqrt{\alpha} x-d(t) \alpha \sqrt{\alpha} \cos \sqrt{\alpha} x \\
\phi_{t}= & \left.4 f_{x x}\right|_{x=0} \frac{\sin \sqrt{\alpha} x}{\sqrt{\alpha}}+\left.4 f_{x}\right|_{x=0} \cos \sqrt{\alpha} x-\left.4 f\right|_{x=0}(\sqrt{\alpha} \sin \sqrt{\alpha} x) \\
& -4 f_{x}+\int_{0}^{x} 4 f\left(x^{\prime}, t\right)\left(\alpha \cos \sqrt{\alpha}\left(x-x^{\prime}\right)\right) d x^{\prime} \\
& +c_{t} \cos \sqrt{\alpha} x+d_{t} \sin \sqrt{\alpha} x,
\end{aligned}
$$


where $f_{t}=-4 f_{x x x}$ has been used for the computation of $\phi_{t}$. Therefore, the differential equation (3.11) equivalently requires

$$
\begin{aligned}
& \left.\frac{4}{\sqrt{\alpha}} f_{x x}\right|_{x=0}-\left.4 \sqrt{\alpha} f\right|_{x=0}+d_{t}=-4 \alpha \sqrt{\alpha} c, \\
& \left.4 f_{x}\right|_{x=0}+c_{t}=4 \alpha \sqrt{\alpha} d .
\end{aligned}
$$

Then it follows that

$$
c_{t t}+16 \alpha^{3} c+h=0,
$$

where the function $h=h(t)$ is defined by

$$
h:=\left.16 \alpha f_{x x}\right|_{x=0}-\left.16 \alpha^{2} f\right|_{x=0}+\left.4 f_{x t}\right|_{x=0} .
$$

Again by Lemma 3.3, we obtain

$$
c(t)=-\int_{0}^{t} \frac{\sin 4 \alpha \sqrt{\alpha}\left(t-t^{\prime}\right)}{4 \alpha \sqrt{\alpha}} h\left(t^{\prime}\right) d t^{\prime}+c_{0} \cos 4 \alpha \sqrt{\alpha} t+d_{0} \sin 4 \alpha \sqrt{\alpha} t,
$$

where $c_{0}$ and $d_{0}$ are arbitrary real constants. Noting that $h$ is given by (3.15), we have

$$
\begin{aligned}
c(t)= & f_{x}(0,0) \frac{\sin 4 \alpha \sqrt{\alpha} t}{\alpha \sqrt{\alpha}}-4 \int_{0}^{t} f_{x}\left(0, t^{\prime}\right) \cos 4 \alpha \sqrt{\alpha}\left(t-t^{\prime}\right) d t^{\prime} \\
& -4 \int_{0}^{t}\left(\frac{1}{\sqrt{\alpha}} f_{x x}-\sqrt{\alpha} f\right)\left(0, t^{\prime}\right) \sin 4 \alpha \sqrt{\alpha}\left(t-t^{\prime}\right) d t^{\prime} \\
& +c_{0} \cos 4 \alpha \sqrt{\alpha} t+d_{0} \sin 4 \alpha \sqrt{\alpha} t,
\end{aligned}
$$

and then by (3.14), we have

$$
\begin{aligned}
d(t)= & f_{x}(0,0) \frac{\cos 4 \alpha \sqrt{\alpha} t}{\alpha \sqrt{\alpha}}+4 \int_{0}^{t} f_{x}\left(0, t^{\prime}\right) \sin 4 \alpha \sqrt{\alpha}\left(t-t^{\prime}\right) d t^{\prime} \\
& -4 \int_{0}^{t}\left(\frac{1}{\sqrt{\alpha}} f_{x x}-\sqrt{\alpha} f\right)\left(0, t^{\prime}\right) \cos 4 \alpha \sqrt{\alpha}\left(t-t^{\prime}\right) d t^{\prime} \\
& -c_{0} \sin 4 \alpha \sqrt{\alpha} t+d_{0} \cos 4 \alpha \sqrt{\alpha} t,
\end{aligned}
$$

where $c_{0}$ and $d_{0}$ are independent of $x$ and $t$.

Finally, summing up, we have the following theorem on the general solution of the system of differential equations (3.10) and (3.11).

Theorem 3.4. Let $f=f(x, t)$ be a given function satisfying the compatibility condition $f_{t}=-4 f_{x x x}$, and $\alpha$ a positive constant. Then the system of differential equations (3.11) and (3.10) has the general solution given by (3.12)) with $c(t)$ and $d(t)$ being shown by 3.16) and (3.17), respectively.

3.2. The case of complex eigenvalues. Let us now consider the second representative system (3.2). To present its solution formula, we first solve the following coupled system of second-order, non-homogeneous ordinary differential equations

$$
\left\{\begin{array}{l}
-\phi_{1, x x}=\alpha \phi_{1}-\beta \phi_{2}+f_{1}, \\
-\phi_{2, x x}=\beta \phi_{1}+\alpha \phi_{2}+f_{2},
\end{array}\right.
$$


where $\alpha$ and $\beta>0$ are real constants, and $f_{1}$ and $f_{2}$ are two given functions of $x$. Since $\beta>0$, its coefficient matrix

$$
A=\left[\begin{array}{cc}
\alpha & -\beta \\
\beta & \alpha
\end{array}\right]
$$

has two complex eigenvalues $\lambda^{ \pm}=\alpha \pm \beta i$.

Theorem 3.5. The coupled non-homogenous system of second-order ordinary differential equations (3.18) has the general solution

$$
\begin{aligned}
\phi_{1} & =\phi_{1}^{h}+\phi_{1}^{s}, \phi_{2}=\phi_{2}^{h}+\phi_{2}^{s} \\
\phi_{1}^{h} & =\left(D_{1} \cos \delta x+D_{2} \sin \delta x\right) e^{\Delta x}+\left(D_{3} \cos \delta x+D_{4} \sin \delta x\right) e^{-\Delta x} \\
\phi_{2}^{h} & =\left(-D_{2} \cos \delta x+D_{1} \sin \delta x\right) e^{\Delta x}+\left(-D_{4} \cos \delta x+D_{3} \sin \delta x\right) e^{-\Delta x} \\
\phi_{1}^{s} & =-\frac{1}{\Delta} \int_{0}^{x}\left[f_{1}(y) \cos \delta(x-y)-f_{2}(y) \sin \delta(x-y)\right] \sinh \Delta(x-y) d y \\
\phi_{2}^{s} & =-\frac{1}{\Delta} \int_{0}^{x}\left[f_{1}(y) \sin \delta(x-y)+f_{2}(y) \cos \delta(x-y)\right] \sinh \Delta(x-y) d y,
\end{aligned}
$$

where two constants $\Delta$ and $\delta$ are given by

$$
\Delta=\sqrt{\frac{\sqrt{\alpha^{2}+\beta^{2}}-\alpha}{2}}, \delta=\sqrt{\frac{\sqrt{\alpha^{2}+\beta^{2}}+\alpha}{2}},
$$

and $D_{i}, 1 \leq i \leq 4$, are arbitrary real constants.

Proof. First let us consider the homogenous case of (3.18), i.e., the case of (3.18) with $f_{1}=f_{2}=0$. Therefore, we have

$$
\phi_{2}=\frac{1}{\beta} \phi_{1, x x}+\frac{\alpha}{\beta} \phi_{1} .
$$

Then replacing $\phi_{2}$ with this expression in the second equation of the homogenous case leads to the following fourth-order differential equation

$$
\phi_{1, x x x x}+2 \alpha \phi_{1, x x}+\left(\alpha^{2}+\beta^{2}\right) \phi_{1}=0 .
$$

Its characteristic polynomial

$$
p(\lambda)=\lambda^{4}+2 \alpha \lambda^{2}+\left(\alpha^{2}+\beta^{2}\right)=0
$$

has four complex roots

$$
\lambda_{1}^{ \pm}=\Delta \pm \delta \sqrt{-1}, \lambda_{2}^{ \pm}=-\Delta \pm \delta \sqrt{-1},
$$

where $\Delta$ and $\delta$ are defined by (3.24). Therefore, we have the general solution $\phi_{1}^{h}$ and $\phi_{2}^{h}$, as given in the theorem, for the homogeneous case of (3.18).

To construct a special solution, $\phi_{1}^{s}$ and $\phi_{2}^{s}$, for the non-homogenous case of (3.18), we adopt the method of variation of parameters for $D_{i}, 1 \leq i \leq 4$, and thus assume that

$$
\left\{\begin{array}{l}
\phi_{1}^{s}=\left(D_{1} \cos \delta x+D_{2} \sin \delta x\right) \mathrm{e}^{\Delta x}+\left(D_{3} \cos \delta x+D_{4} \sin \delta x\right) \mathrm{e}^{-\Delta x}, \\
\phi_{2}^{s}=\left(-D_{2} \cos \delta x+D_{1} \sin \delta x\right) \mathrm{e}^{\Delta x}+\left(-D_{4} \cos \delta x+D_{3} \sin \delta x\right) \mathrm{e}^{-\Delta x}
\end{array}\right.
$$


where $D_{i}, 1 \leq i \leq 4$, are viewed as functions of $x$. Then we can make the following computation:

$$
\begin{aligned}
\phi_{1, x}^{s}= & {\left[\left(\Delta D_{1}+\delta D_{2}\right) \cos \delta x+\left(\Delta D_{2}-\delta D_{1}\right) \sin \delta x\right] \mathrm{e}^{\Delta x} } \\
& \left.+\left(\delta D_{4}-\Delta D_{3}\right) \cos \delta x-\left(\delta D_{3}+\Delta D_{4}\right) \sin \delta x\right] \mathrm{e}^{-\Delta x} \\
\phi_{1, x x}^{s}= & {\left[\Delta\left(\Delta D_{1}+\delta D_{2}\right)+\delta\left(\Delta D_{2}-\delta D_{1}\right)\right](\cos \delta x) \mathrm{e}^{\Delta x} } \\
& +\left[\Delta\left(\Delta D_{2}-\delta D_{1}\right)-\delta\left(\Delta D_{1}+\delta D_{2}\right)\right](\sin \delta x) \mathrm{e}^{\Delta x} \\
& +\left[-\delta\left(\delta D_{3}+\Delta D_{4}\right)-\Delta\left(\delta D_{4}-\Delta D_{3}\right)\right](\cos \delta x) \mathrm{e}^{-\Delta x} \\
& +\left[-\delta\left(\delta D_{4}-\Delta D_{3}\right)+\Delta\left(\delta D_{3}+\Delta D_{4}\right)\right](\sin \delta x) \mathrm{e}^{-\Delta x} \\
= & \left(\alpha D_{1}+\beta D_{2}\right)(\cos \delta x) \mathrm{e}^{\Delta x}+\left(-\beta D_{1}+\alpha D_{2}\right)(\sin \delta x) \mathrm{e}^{\Delta x} \\
& +\left(\alpha D_{3}-\beta D_{4}\right)(\cos \delta x) \mathrm{e}^{-\Delta x}+\left(\beta D_{3}+\alpha D_{4}\right)(\sin \delta x) \mathrm{e}^{-\Delta x} \\
:= & \left(\tilde{D}_{1} \cos \delta x+\tilde{D}_{2} \sin \delta x\right) \mathrm{e}^{\Delta x}+\left(\tilde{D}_{3} \cos \delta x+\tilde{D}_{4} \sin \delta x\right) \mathrm{e}^{-\Delta x}, \\
\phi_{2, x}^{s}= & {\left[\left(-\Delta D_{2}+\delta D_{1}\right) \cos \delta x+\left(\Delta D_{1}+\delta D_{2}\right) \sin \delta x\right] \mathrm{e}^{\Delta x} } \\
& \left.+\left(\delta D_{3}+\Delta D_{4}\right) \cos \delta x+\left(\delta D_{4}-\Delta D_{3}\right) \sin \delta x\right] \mathrm{e}^{-\Delta x}, \\
\phi_{2, x x}^{s}= & \left(-\alpha D_{2}+\beta D_{1}\right)(\cos \delta x) \mathrm{e}^{\Delta x}+\left(\beta D_{2}+\alpha D_{1}\right)(\sin \delta x) \mathrm{e}^{\Delta x} \\
& -\left(\alpha D_{4}+\beta D_{3}\right)(\cos \delta x) \mathrm{e}^{-\Delta x}-\left(\beta D_{4}-\alpha D_{3}\right)(\sin \delta x) \mathrm{e}^{-\Delta x}
\end{aligned}
$$

In the above computation, the method of variation of parameters required that

$$
\begin{aligned}
& \left(D_{1, x} \cos \delta x+D_{2, x} \sin \delta x\right) \mathrm{e}^{\Delta x}+\left(D_{3, x} \cos \delta x+D_{4, x} \sin \delta x\right) \mathrm{e}^{-\Delta x}=0, \\
& {\left[\left(\Delta D_{1, x}+\delta D_{2, x}\right) \cos \delta x+\left(\Delta D_{2, x}-\delta D_{1, x}\right) \sin \delta x\right] \mathrm{e}^{\Delta x}} \\
& \left.+\left(\delta D_{4, x}-\Delta D_{3, x}\right) \cos \delta x-\left(\delta D_{3, x}+\Delta D_{4, x}\right) \sin \delta x\right] \mathrm{e}^{-\Delta x}=-f_{1}, \\
& \left(-D_{2, x} \cos \delta x+D_{1, x} \sin \delta x\right) \mathrm{e}^{\Delta x}-\left(D_{4, x} \cos \delta x-D_{3, x} \sin \delta x\right) \mathrm{e}^{-\Delta x}=0, \\
& {\left[\left(-\Delta D_{2, x}+\delta D_{1, x}\right) \cos \delta x+\left(\Delta D_{1, x}+\delta D_{2, x}\right) \sin \delta x\right] \mathrm{e}^{\Delta x}} \\
& \left.+\left(\delta D_{3, x}+\Delta D_{4, x}\right) \cos \delta x+\left(\delta D_{4, x}-\Delta D_{3, x}\right) \sin \delta x\right] \mathrm{e}^{-\Delta x}=-f_{2} .
\end{aligned}
$$

Solving this linear system for $D_{i, x}$ leads to the expressions:

$$
\begin{aligned}
& D_{1, x}=-\frac{1}{2 \Delta}\left(f_{1} \cos \delta x+f_{2} \sin \delta x\right) \mathrm{e}^{-\Delta x}, \\
& D_{2, x}=-\frac{1}{2 \Delta}\left(f_{1} \sin \delta x-f_{2} \cos \delta x\right) \mathrm{e}^{-\Delta x}, \\
& D_{3, x}=\frac{1}{2 \Delta}\left(f_{1} \cos \delta x+f_{2} \sin \delta x\right) \mathrm{e}^{-\Delta x}=-D_{1, x}, \\
& D_{4, x}=\frac{1}{2 \Delta}\left(f_{1} \sin \delta x-f_{2} \cos \delta x\right) \mathrm{e}^{-\Delta x}=-D_{2, x} .
\end{aligned}
$$

Then integrating them to obtain the expressions for $D_{i}$ and inserting the resultant expressions of $D_{i}$ into (3.27) yield the special solution, $\phi_{1}^{s}$ and $\phi_{2}^{s}$, given in the theorem. The proof is finished.

Let us then present the solution formula for the second representative system (3.2), which can be used to generate a new type of Wronskian solutions to the KdV equation.

Theorem 3.6. Assume that $f_{1}=f_{1}(x, t)$ and $f_{2}=f_{2}(x, t)$ are two given functions satisfying the compatibility conditions $f_{1, t}=-4 f_{1, x x x}$ and $f_{2, t}=-4 f_{2, x x x}$. Then 
the general solution to the system of differential equations (3.2) is given by (3.19)(3.23) with the coefficients $D_{i}=D_{i}(t), 1 \leq i \leq 4$, being determined by

$$
\begin{aligned}
& {\left[\begin{array}{l}
D_{1} \\
D_{2}
\end{array}\right]=e^{\tilde{\alpha} t}\left[\begin{array}{cc}
\cos \tilde{\beta} t & -\sin \tilde{\beta} t \\
\sin \tilde{\beta} s & \cos \tilde{\beta} t
\end{array}\right]\left[\begin{array}{c}
D_{10} \\
D_{20}
\end{array}\right]} \\
& +\int_{0}^{t} e^{\tilde{\alpha}(t-s)}\left[\begin{array}{cc}
\cos \tilde{\beta}(t-s) & -\sin \tilde{\beta}(t-s) \\
\sin \tilde{\beta}(t-s) & \cos \tilde{\beta}(t-s)
\end{array}\right]\left[\begin{array}{c}
p_{1}(s) \\
p_{2}(s)
\end{array}\right] d s, \\
& {\left[\begin{array}{c}
D_{3} \\
D_{4}
\end{array}\right]=e^{-\tilde{\alpha} t}\left[\begin{array}{cc}
\cos \tilde{\beta} t & -\sin \tilde{\beta} t \\
\sin \tilde{\beta} s & \cos \tilde{\beta} t
\end{array}\right]\left[\begin{array}{c}
D_{30} \\
D_{40}
\end{array}\right]} \\
& +\int_{0}^{t} e^{-\tilde{\alpha}(t-s)}\left[\begin{array}{cc}
\cos \tilde{\beta}(t-s) & -\sin \tilde{\beta}(t-s) \\
\sin \tilde{\beta}(t-s) & \cos \tilde{\beta}(t-s)
\end{array}\right]\left[\begin{array}{l}
p_{3}(s) \\
p_{4}(s)
\end{array}\right] d s,
\end{aligned}
$$

where $D_{i 0}, 1 \leq i \leq 4$, are arbitrary real constants, and two constants $\tilde{\alpha}$ and $\tilde{\beta}$ are given by

$$
\tilde{\alpha}=-4 \Delta\left(\sqrt{\alpha^{2}+\beta^{2}}+2 \alpha\right), \tilde{\beta}=4 \delta\left(\sqrt{\alpha^{2}+\beta^{2}}-2 \alpha\right),
$$

and four functions, $p_{i}, 1 \leq i \leq 4$, are defined by

$$
\begin{aligned}
& p_{1}(t)=\left.\frac{2}{\Delta} f_{1, x x}\right|_{x=0}+\left.2 f_{1, x}\right|_{x=0}+\left.\frac{2 \alpha}{\Delta} f_{1}\right|_{x=0}-\left.\frac{2 \delta}{\Delta} f_{2, x}\right|_{x=0}-\left.4 \delta f_{2}\right|_{x=0}, \\
& p_{2}(t)=-\left.\frac{2 \delta}{\Delta} f_{1, x}\right|_{x=0}-\left.4 \delta f_{1}\right|_{x=0}-\left.\frac{2}{\Delta} f_{2, x x}\right|_{x=0}-\left.2 f_{2, x}\right|_{x=0}-\left.\frac{2 \alpha}{\Delta} f_{2}\right|_{x=0}, \\
& p_{3}(t)=-\left.\frac{2}{\Delta} f_{1, x x}\right|_{x=0}+\left.2 f_{1, x}\right|_{x=0}-\left.\frac{2 \alpha}{\Delta} f_{1}\right|_{x=0}+\left.\frac{2 \delta}{\Delta} f_{2, x}\right|_{x=0}-\left.4 \delta f_{2}\right|_{x=0}, \\
& p_{4}(t)=\left.\frac{2 \delta}{\Delta} f_{1, x}\right|_{x=0}-\left.4 \delta f_{1}\right|_{x=0}+\left.\frac{2}{\Delta} f_{2, x x}\right|_{x=0}-\left.2 f_{2, x}\right|_{x=0}+\left.\frac{2 \alpha}{\Delta} f_{2}\right|_{x=0},
\end{aligned}
$$

with $\Delta$ and $\delta$ being defined by (3.24).

Proof. Note that now $f_{1}=f_{1}(x, t)$ and $f_{2}=f_{2}(x, t)$ are functions of two variables $x$ and $t$. But in the solution formula (3.22) and (3.23), $t$ is the dummy variable. Let us first compute that

$$
\begin{aligned}
\phi_{1, t}^{h}= & \left(D_{1, t} \cos \delta x+D_{2, t} \sin \delta x\right) \mathrm{e}^{\Delta x}+\left(D_{3, t} \cos \delta x+D_{4, t} \sin \delta x\right) \mathrm{e}^{-\Delta x} \\
\phi_{1, t}^{s}= & -\int_{0}^{x}\left[g_{1}(x-y) f_{1, t}(y, t)+g_{2}(x-y) f_{2, t}(y, t)\right] d y \\
= & 4 \int_{0}^{x}\left[g_{1}(x-y) f_{1, y y y}(y, t)+g_{2}(x-y) f_{2, y y y}(y, t)\right] d y \\
= & -4 g_{1}(x)\left(\left.f_{1, x x}\right|_{x=0}\right)-4 g_{1, x}(x)\left(\left.f_{1, x}\right|_{x=0}\right)-4 g_{1, x x}(x)\left(\left.f_{1}\right|_{x=0}\right) \\
& -4 g_{2}(x)\left(\left.f_{2, x x}\right|_{x=0}\right)-4 g_{2, x}(x)\left(\left.f_{2, x}\right|_{x=0}\right)-4 g_{2, x x}(x)\left(\left.f_{2}\right|_{x=0}\right)-4 \phi_{1, x x x}^{s},
\end{aligned}
$$

where

$$
g_{1}=\frac{1}{\Delta}(\cos \delta x)(\sinh \Delta x), g_{2}=-\frac{1}{\Delta}(\sin \delta x)(\sinh \Delta x) .
$$

Second, based on (3.28) and (3.29), we have

$$
\begin{aligned}
\phi_{1, x x x}^{h}= & {\left[\left(\Delta \tilde{D}_{1}+\delta \tilde{D}_{2}\right) \cos \delta x+\left(\Delta \tilde{D}_{2}-\delta \tilde{D}_{1}\right) \sin \delta x\right] \mathrm{e}^{\Delta x} } \\
& +\left[\left(\delta \tilde{D}_{4}-\Delta \tilde{D}_{3}\right) \cos \delta x-\left(\delta \tilde{D}_{3}+\Delta \tilde{D}_{4}\right) \sin \delta x\right] \mathrm{e}^{-\Delta x},
\end{aligned}
$$


and a direct computation gives rise to

$$
\begin{aligned}
& g_{1, x}(x)=\frac{1}{2}(\cos \delta x)\left(\mathrm{e}^{\Delta x}+\mathrm{e}^{-\Delta x}\right)-\frac{\delta}{2 \Delta}(\sin \delta x)\left(\mathrm{e}^{\Delta x}-\mathrm{e}^{-\Delta x}\right), \\
& g_{1, x x}(x)=\left(-\delta \sin \delta x+\frac{\alpha}{2 \Delta} \cos \delta x\right) \mathrm{e}^{\Delta x}-\left(\delta \sin \delta x+\frac{\alpha}{2 \Delta} \cos \delta x\right) \mathrm{e}^{-\Delta x}, \\
& g_{2, x}(x)=-\frac{1}{2}(\sin \delta x)\left(\mathrm{e}^{\Delta x}+\mathrm{e}^{-\Delta x}\right)-\frac{\delta}{2 \Delta}(\cos \delta x)\left(\mathrm{e}^{\Delta x}-\mathrm{e}^{-\Delta x}\right), \\
& g_{2, x x}(x)=-\left(\delta \cos \delta x+\frac{\alpha}{2 \Delta} \sin \delta x\right) \mathrm{e}^{\Delta x}-\left(\delta \cos \delta x-\frac{\alpha}{2 \Delta} \sin \delta x\right) \mathrm{e}^{-\Delta x} .
\end{aligned}
$$

Now a comparison of coefficients of four functionally independent terms

$$
(\cos \delta x) \mathrm{e}^{\Delta x},(\sin \delta x) \mathrm{e}^{\Delta x},(\cos \delta x) \mathrm{e}^{-\Delta x},(\sin \delta x) \mathrm{e}^{-\Delta x}
$$

in the equation $\phi_{1, t}=-4 \phi_{1, x x x}$, i.e., $\phi_{1, t}^{h}+\phi_{1, t}^{s}=-4 \phi_{1, x x x}^{h}-4 \phi_{1, x x x}^{s}$, yields that

$$
\begin{aligned}
D_{1, t} & =-4\left(\Delta \tilde{D}_{1}+\delta \tilde{D}_{2}\right)+p_{1}(t) \\
& =4\left(3 \Delta \delta^{2}-\Delta^{3}\right) D_{1}-4\left(3 \Delta^{2} \delta-\delta^{3}\right) D_{2}+p_{1}(t) \\
& =\tilde{\alpha} D_{1}-\tilde{\beta} D_{2}+p_{1}(t), \\
D_{2, t} & =-4\left(\Delta \tilde{D}_{2}-\delta \tilde{D}_{1}\right)+p_{2}(t) \\
& =4\left(3 \Delta^{2} \delta-\delta^{3}\right) D_{1}+4\left(3 \Delta \delta^{2}-\Delta^{3}\right) D_{2}+p_{2}(t) \\
& =\tilde{\beta} D_{1}+\tilde{\alpha} D_{2}+p_{2}(t), \\
D_{3, t} & =-4\left(\delta \tilde{D}_{4}-\Delta \tilde{D}_{3}\right)+p_{3}(t) \\
& =-4\left(3 \Delta \delta^{2}-\Delta^{3}\right) D_{3}-4\left(3 \Delta^{2} \delta-\delta^{3}\right) D_{4}+p_{3}(t) \\
& =-\tilde{\alpha} D_{3}-\tilde{\beta} D_{4}+p_{3}(t), \\
D_{4, t} & =-4\left(-\delta \tilde{D}_{3}-\Delta \tilde{D}_{4}\right)+p_{4}(t) \\
& =4\left(3 \Delta^{2} \delta-\delta^{3}\right) D_{3}-4\left(3 \Delta \delta^{2}-\Delta^{3}\right) D_{4}+p_{4}(t) \\
& =\tilde{\beta} D_{3}-\tilde{\alpha} D_{4}+p_{4}(t) .
\end{aligned}
$$

These are two linear systems of first-order ordinary differential equations. One is for $D_{1}$ and $D_{2}$ and the other is for $D_{3}$ and $D_{4}$. Solving these two coupled linear systems for $D_{i}, 1 \leq i \leq 4$, we obtain the general solution given by (3.32) and (3.33) in the theorem. For example, we rewrite (3.35) and (3.36) as

$$
\left[\begin{array}{c}
D_{1, t} \\
D_{2, t}
\end{array}\right]=\tilde{A}\left[\begin{array}{c}
D_{1} \\
D_{2}
\end{array}\right]+\left[\begin{array}{c}
p_{1} \\
p_{2}
\end{array}\right], \tilde{A}=\left[\begin{array}{cc}
\tilde{\alpha} & -\tilde{\beta} \\
\tilde{\beta} & \tilde{\alpha}
\end{array}\right] .
$$

Then it follows that

$$
\left[\begin{array}{l}
D_{1} \\
D_{2}
\end{array}\right]=\mathrm{e}^{\tilde{A} t}\left[\begin{array}{c}
D_{10} \\
D_{20}
\end{array}\right]+\mathrm{e}^{\tilde{A} t} \int_{0}^{t} \mathrm{e}^{-\tilde{A} s}\left[\begin{array}{c}
p_{1}(s) \\
p_{2}(s)
\end{array}\right] d s .
$$

Since $\tilde{A}$ has two complex eigenvalues

$$
\lambda^{ \pm}=\tilde{\alpha} \pm \tilde{\beta} \sqrt{-1},
$$

the evolution operator $\mathrm{e}^{\tilde{A} t}$ reads as [HS, $\mathrm{AP}$.

$$
\mathrm{e}^{\tilde{A} t}=\mathrm{e}^{\tilde{\alpha} t}\left[\begin{array}{cc}
\cos \tilde{\beta} t & -\sin \tilde{\beta} t \\
\sin \tilde{\beta} t & \cos \tilde{\beta} t
\end{array}\right] .
$$


The derivation of the formulas for $D_{3}$ and $D_{4}$ is completely similar. Note that the other equation $\phi_{2, t}=-4 \phi_{2, x x x}$ is automatically satisfied, once $D_{i}, 1 \leq i \leq 4$, are determined as above. The proof is finished.

\section{WRONSKIAN SOLUTIONS}

In principle, we can construct general solutions associated with two types of Jordan blocks of the coefficient matrix through the solution formulas established in the previous section. In what follows, we present a few special but interesting Wronskian solutions, rather than general ones which are too complicated and lengthy to present.

4.1. Rational solutions. Let us start with

$$
\psi(\eta)=\mathrm{e}^{\eta x-4 \eta^{3} t}-\mathrm{e}^{-\eta x+4 \eta^{3} t}=2 \sinh \left(\eta x-4 \eta^{3} t\right),
$$

where $\eta$ is a real constant. It is easy to see that this function $\psi(\eta)$ solves

$$
\psi_{x x}=\eta^{2} \psi, \psi_{t}=-4 \psi_{x x x} .
$$

Upon expanding

$$
\psi(\eta)=\sum_{i=0}^{\infty} \phi_{i} \eta^{2 i+1}
$$

from (4.2) we obtain

$$
\phi_{0, x x}=0, \phi_{i+1, x x}=\phi_{i}, \phi_{i, t}=-4 \phi_{i, x x x}, i \geq 0 .
$$

The expressions of $\phi_{i}$ can be calculated through the series expansion of the hyperbolic function $\sinh x$ :

$$
\phi_{i}=\sum_{j=0}^{[(2 i+1) / 3]} \frac{(-4)^{j}}{(2 i-3 j+1) ! j !} x^{2 i-3 j+1} t^{j}, i \geq 0 .
$$

Then for each $k \geq 1$, the associated Wronskian solution to the KdV equation (1.1) is given by

$$
u=-2 \partial_{x}^{2} \ln W\left(\phi_{0}, \phi_{1}, \cdots, \phi_{k-1}\right) .
$$

Obviously, this solution is rational and it corresponds to the following Jordan block:

$$
\left[\begin{array}{cccc}
0 & & & 0 \\
1 & 0 & & \\
& \ddots & \ddots & \\
0 & & 1 & 0
\end{array}\right]_{k \times k} .
$$

We call it a rational solution of order $k-1$. This type of solutions was also discussed in $[\mathrm{ASa}, \mathrm{SHR}, \mathrm{AM}]$ and obtained by Freeman and Nimmo [FN] by taking the limit $\eta_{i} \rightarrow 0$ of the multi-soliton solution. Two rational solutions of lower-order are

$$
\begin{aligned}
& u=-2 \partial_{x}^{2} \ln W\left(\phi_{0}\right)=-2 \partial_{x}^{2} \ln W(x)=\frac{2}{x^{2}} \\
& u=-2 \partial_{x}^{2} \ln W\left(\phi_{0}, \phi_{1}\right)=-2 \partial_{x}^{2} \ln W\left(x, \frac{1}{6} x^{3}-4 t\right)=\frac{6 x^{4}-144 x t}{\left(x^{3}+12 t\right)^{2}} .
\end{aligned}
$$


4.2. Solitons, positons and negatons. For a nonzero real eigenvalue $\lambda_{i}$, we start from the eigenfunction $\phi_{i}\left(\lambda_{i}\right)$ determined by

$$
-\left(\phi_{i}\left(\lambda_{i}\right)\right)_{x x}=\lambda_{i} \phi_{i}\left(\lambda_{i}\right),\left(\phi_{i}\left(\lambda_{i}\right)\right)_{t}=-4\left(\phi_{i}\left(\lambda_{i}\right)\right)_{x x x} .
$$

General solutions to this system in two cases of $\lambda_{i}>0$ and $\lambda_{i}<0$ are

$$
\begin{aligned}
& \phi_{i}\left(\lambda_{i}\right)=C_{1 i} \sin \left(\eta_{i} x+4 \eta_{i}^{3} t\right)+C_{2 i} \cos \left(\eta_{i} x+4 \eta_{i}^{3} t\right), \eta_{i}=\sqrt{\lambda_{i}}, \\
& \phi_{i}\left(\lambda_{i}\right)=C_{1 i} \sinh \left(\eta_{i} x-4 \eta_{i}^{3} t\right)+C_{2 i} \cosh \left(\eta_{i} x-4 \eta_{i}^{3} t\right), \eta_{i}=\sqrt{-\lambda_{i}},
\end{aligned}
$$

respectively, where $C_{1 i}$ and $C_{2 i}$ are arbitrary real constants. By an inspection, we find that

$$
-\left[\begin{array}{c}
\phi_{i}\left(\lambda_{i}\right) \\
\frac{1}{1 !} \partial_{\lambda_{i}} \phi_{i}\left(\lambda_{i}\right) \\
\vdots \\
\frac{1}{\left(k_{i}-1\right) !} \partial_{\lambda_{i}}^{k_{i}-1} \phi_{i}\left(\lambda_{i}\right)
\end{array}\right]_{x x}=\left[\begin{array}{cccc}
\lambda_{i} & & & 0 \\
1 & \lambda_{i} & & \\
& \ddots & \ddots & \\
0 & & 1 & \lambda_{i}
\end{array}\right]_{k_{i} \times k_{i}}\left[\begin{array}{c}
\phi_{i}\left(\lambda_{i}\right) \\
\frac{1}{1 !} \partial_{\lambda_{i}} \phi_{i}\left(\lambda_{i}\right) \\
\vdots \\
\frac{1}{\left(k_{i}-1\right) !} \partial_{\lambda_{i}}^{k_{i}-1} \phi_{i}\left(\lambda_{i}\right)
\end{array}\right],
$$

and

$$
\left(\frac{1}{j !} \partial_{\lambda_{i}}^{j} \phi_{i}\left(\lambda_{i}\right)\right)_{t}=-4\left(\frac{1}{j !} \partial_{\lambda_{i}}^{j} \phi_{i}\left(\lambda_{i}\right)\right)_{x x x}, 0 \leq j \leq k_{i}-1,
$$

where $\partial_{\lambda_{i}}$ denotes the derivative with respect to $\lambda_{i}$ and $k_{i}$ is an arbitrary nonnegative integer. Therefore, through this set of eigenfunctions, we obtain a Wronskian solution to the KdV equation (1.1):

$$
u=-2 \partial_{x}^{2} \ln W\left(\phi_{i}\left(\lambda_{i}\right), \frac{1}{1 !} \partial_{\lambda_{i}} \phi_{i}\left(\lambda_{i}\right), \cdots, \frac{1}{\left(k_{i}-1\right) !} \partial_{\lambda_{i}}^{k_{i}-1} \phi_{i}\left(\lambda_{i}\right)\right),
$$

which corresponds to the first type of Jordan blocks with a nonzero real eigenvalue.

When $\lambda_{i}>0$, we get positon solutions [Mat], and when $\lambda_{i}<0$, we get negaton solutions [RSK]. A more general positon or negaton can be obtained by combining $n$ sets of eigenfunctions associated with different $\lambda_{i}>0$ or different $\lambda_{i}<0$ :

$$
\begin{aligned}
u= & -2 \partial_{x}^{2} \ln W\left(\phi_{1}\left(\lambda_{1}\right), \cdots, \frac{1}{\left(k_{1}-1\right) !} \partial_{\lambda_{1}}^{k_{1}-1} \phi_{1}\left(\lambda_{1}\right) ;\right. \\
& \left.\cdots ; \phi_{n}\left(\lambda_{n}\right), \cdots, \frac{1}{\left(k_{n}-1\right) !} \partial_{\lambda_{n}}^{k_{n}-1} \phi_{n}\left(\lambda_{n}\right)\right) .
\end{aligned}
$$

This solution is called an $n$-position or $n$-negaton of order $\left(k_{1}-1, k_{2}-1, \cdots\right.$, $k_{n}-1$ ), to reflect the number of different eigenvalues and the orders of derivatives of eigenfunctions. If $k_{i}=1,1 \leq i \leq n$, we simply say that it is an $n$-position or $n$-negaton.

An $n$-soliton solution is a special $n$-negaton:

$$
u=-2 \partial_{x}^{2} \ln \left(\phi_{1}, \phi_{2}, \cdots, \phi_{n}\right)
$$

with $\phi_{i}$ being given by

$$
\begin{gathered}
\phi_{i}=\cosh \left(\eta_{i} x-4 \eta_{i}^{3} t+\gamma_{i}\right), \quad i \text { odd, } \\
\phi_{i}=\sinh \left(\eta_{i} x-4 \eta_{i}^{3} t+\gamma_{i}\right), \quad i \text { even, }
\end{gathered}
$$

where $0<\eta_{1}<\eta_{2}<\cdots<\eta_{n}$ and $\gamma_{i}, 1 \leq i \leq n$, are arbitrary real constants. There are other representations of multi-soliton solutions to the KdV equation (see, for 
example, $[\mathrm{H}[\mathrm{BS}]$ ). Two kinds of special positons of order $k$ are

$$
\begin{aligned}
& u=-2 \partial_{x}^{2} \ln W\left(\phi, \partial_{\lambda} \phi, \cdots, \partial_{\lambda}^{k-1} \phi\right), \phi=\cos \left(\eta x+4 \eta^{3} t+\gamma(\eta)\right), \\
& u=-2 \partial_{x}^{2} \ln W\left(\phi, \partial_{\lambda} \phi, \cdots, \partial_{\lambda}^{k-1} \phi\right), \phi=\sin \left(\eta x+4 \eta^{3} t+\gamma(\eta)\right),
\end{aligned}
$$

where $\lambda>0, \eta=\sqrt{\lambda}$ and $\gamma$ is an arbitrary function of $\eta$. But these two kinds of positons are equivalent to each other, due to the existence of the arbitrary function $\gamma$. Similarly, two kinds of special negatons of order $k$ are

$$
\begin{aligned}
& u=-2 \partial_{x}^{2} \ln W\left(\phi, \partial_{\lambda} \phi, \cdots, \partial_{\lambda}^{k-1} \phi\right), \phi=\cosh \left(\eta x-4 \eta^{3} t+\gamma(\eta)\right), \\
& u=-2 \partial_{x}^{2} \ln W\left(\phi, \partial_{\lambda} \phi, \cdots, \partial_{\lambda}^{k-1} \phi\right), \phi=\sinh \left(\eta x-4 \eta^{3} t+\gamma(\eta)\right),
\end{aligned}
$$

where $\lambda<0, \eta=\sqrt{-\lambda}$ and $\gamma$ is an arbitrary function of $\eta$. These solutions exhibited above were also discussed in a slightly different way in [APP, Mat, RSK]. The following are solitons, positons and negatons of lower-order:

$$
\begin{aligned}
u & =-2 \partial_{x}^{2} \ln \left(\cosh \left(\eta x-4 \eta^{3} t+\gamma\right)\right)=\frac{-2 \eta^{2}}{\cosh ^{2}\left(\eta x-4 \eta^{3} t+\gamma\right)}, \\
u & =-2 \partial_{x}^{2} \ln \left(\cos \left(\eta x+4 \eta^{3} t+\gamma\right)\right)=\frac{2 \eta^{2}}{\cos ^{2}\left(\eta x+4 \eta^{3} t+\gamma\right)}, \\
u & =-2 \partial_{x}^{2} \ln \left(\sinh \left(\eta x-4 \eta^{3} t+\gamma\right)\right)=\frac{2 \eta^{2}}{\sinh ^{2}\left(\eta x-4 \eta^{3} t+\gamma\right)} ; \\
u & =-2 \partial_{x}^{2} \ln W\left(\cosh \left(\eta_{1} x-4 \eta_{1}^{3} t+\gamma_{1}\right), \sinh \left(\eta_{2} x-4 \eta_{2}^{3} t+\gamma_{2}\right)\right) \\
& =\frac{4\left(\eta_{1}^{2}-\eta_{2}^{2}\right)\left[\left(\eta_{2}^{2}-\eta_{1}^{2}\right)+\eta_{1}^{2} \cosh 2 \theta_{2}+\eta_{2}^{2} \cosh 2 \theta_{1}\right]}{\left[\left(\eta_{2}-\eta_{1}\right) \cosh \left(\theta_{1}+\theta_{2}\right)+\left(\eta_{2}+\eta_{1}\right) \cosh \left(\theta_{1}-\theta_{2}\right)\right]^{2}} \\
u & =-2 \partial_{x}^{2} \ln W\left(\cos \left(\eta x+4 \eta^{3} t+\gamma\right), \partial_{\lambda} \cos \left(\eta x+4 \eta^{3} t+\gamma\right)\right) \\
& =\frac{16 \eta^{2}\left[2 \cos ^{2}\left(\eta x+4 \eta^{3} t+\gamma\right)+\left(\eta x+12 \eta^{3} t\right) \sin 2\left(\eta x+4 \eta^{3} t+\gamma\right)\right]}{\left[2\left(\eta x+12 \eta^{3} t\right)+\sin 2\left(\eta x+4 \eta^{3} t+\gamma\right)\right]^{2}} \\
u & =-2 \partial_{x}^{2} \ln W\left(\cosh \left(\eta x-4 \eta^{3} t+\gamma\right), \partial_{\lambda} \cosh \left(\eta x-4 \eta^{3} t+\gamma\right)\right) \\
& =\frac{16 \eta^{2}\left[2 \cosh ^{2}\left(\eta x-4 \eta^{3} t+\gamma\right)-\left(\eta x-12 \eta^{3} t\right) \sinh 2\left(\eta x-4 \eta^{3} t+\gamma\right)\right]}{\left[2\left(\eta x-12 \eta^{3} t\right)+\sinh 2\left(\eta x-4 \eta^{3} t+\gamma\right)\right]^{2}} ;
\end{aligned}
$$

where $\eta, \gamma, \eta_{i}$ and $\gamma_{i}$ are arbitrary real constants, and

$$
\theta_{i}=\eta_{i} x-4 \eta_{i}^{3} t+\gamma_{i}, i=1,2 .
$$

Some parts of the graphs of these solutions with $\gamma=\gamma_{i}=0$ are displayed in Figures 1 and 2 .

4.3. Complexitons. For the second type of Jordan blocks of the coefficient matrix, we start from a pair of eigenfunctions $\Phi_{i}\left(\alpha_{i}, \beta_{i}\right)=\left(\phi_{i 1}\left(\alpha_{i}, \beta_{i}\right), \phi_{i 2}\left(\alpha_{i}, \beta_{i}\right)\right)^{T}$ determined by

$$
-\Phi_{i, x x}=A \Phi_{i}, \Phi_{i}=\left[\begin{array}{c}
\phi_{i 1}\left(\alpha_{i}, \beta_{i}\right) \\
\phi_{i 2}\left(\alpha_{i}, \beta_{i}\right)
\end{array}\right], A_{i}=\left[\begin{array}{cc}
\alpha_{i} & -\beta_{i} \\
\beta_{i} & \alpha_{i}
\end{array}\right],
$$

and

$$
\left(\phi_{i j}\left(\alpha_{i}, \beta_{i}\right)\right)_{t}=-4\left(\phi_{i j}\left(\alpha_{i}, \beta_{i}\right)\right)_{x x x}, j=1,2 \text {. }
$$



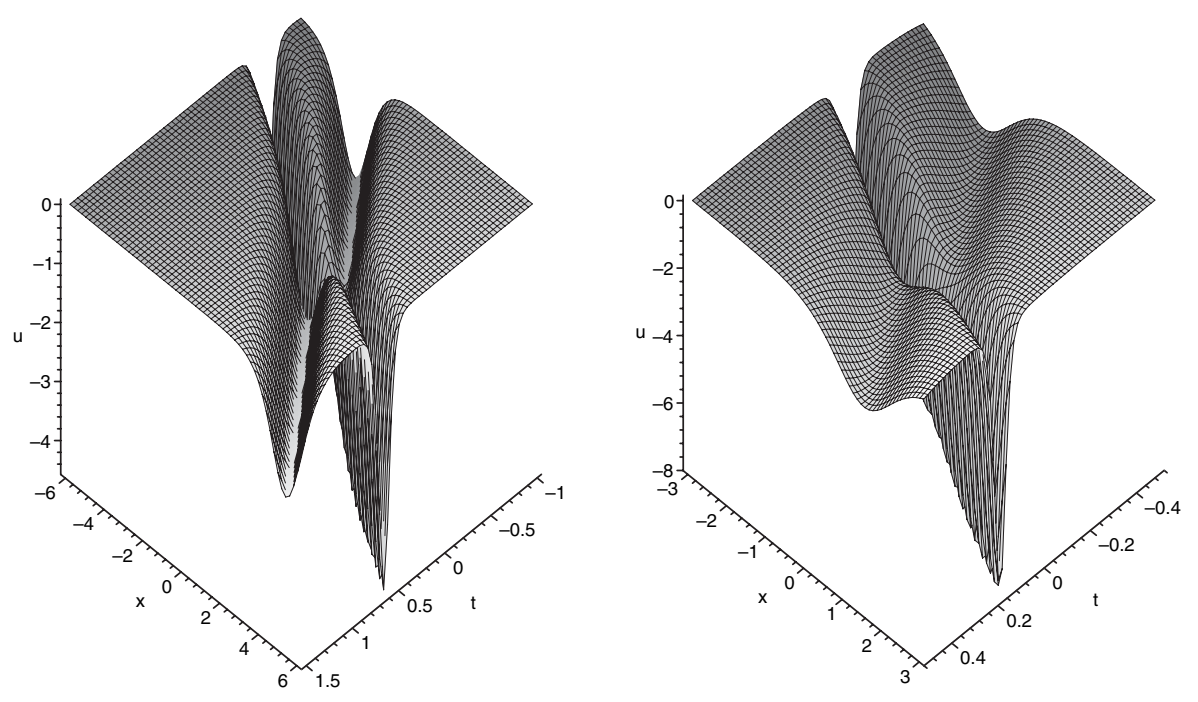

Figure 1. 2-soliton - $\eta_{1}=1, \eta_{2}=1.5$ (left) 2 -soliton $-\eta_{1}=$ $1, \eta_{2}=2$ (right)
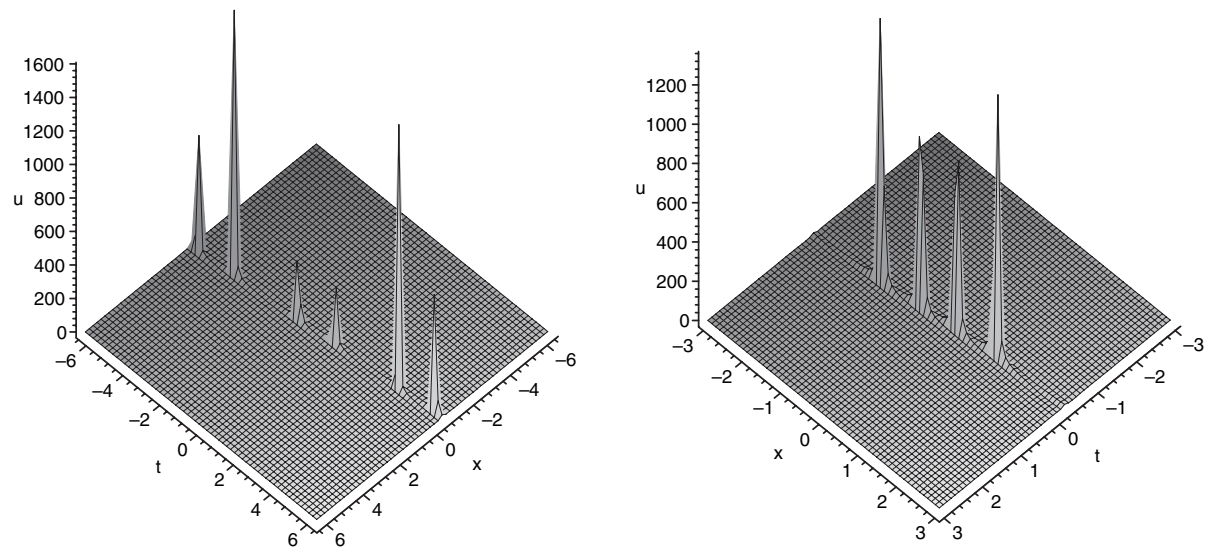

Figure 2. 1-positon of order $1-\lambda=1$ (left) 1 -negaton of order $1-\lambda=-1$ (right)

The coefficient matrix $A_{i}$ has two complex eigenfunctions: $\alpha_{i} \pm \beta_{i} \sqrt{-1}$. By Theorem 3.6, a general solution to the homogenous system of (4.19) and (4.20) is

$$
\begin{aligned}
\phi_{i 1}= & \frac{1}{2}\left[\cos \left(\delta_{i}\left(x-\bar{\beta}_{i} t\right)+\kappa_{1 i}\right)\right] \mathrm{e}^{\Delta_{i}\left(x+\bar{\alpha}_{i} t\right)+\gamma_{1 i}} \\
& +\frac{1}{2}\left[\cos \left(\delta_{i}\left(x-\bar{\beta}_{i} t\right)+\kappa_{2 i}\right)\right] \mathrm{e}^{-\Delta_{i}\left(x+\bar{\alpha}_{i} t\right)-\gamma_{2 i}}, \\
\phi_{i 2}= & \frac{1}{2}\left[\sin \left(\delta_{i}\left(x-\bar{\beta}_{i} t\right)+\kappa_{1 i}\right)\right] \mathrm{e}^{\Delta_{i}\left(x+\bar{\alpha}_{i} t\right)+\gamma_{1 i}} \\
& -\frac{1}{2}\left[\sin \left(\delta_{i}\left(x-\bar{\beta}_{i} t\right)+\kappa_{2 i}\right)\right] \mathrm{e}^{-\Delta_{i}\left(x+\bar{\alpha}_{i} t\right)-\gamma_{2 i}},
\end{aligned}
$$


where $\kappa_{j i}$ and $\gamma_{j i}$ are arbitrary real constants and

$$
\begin{aligned}
& \Delta_{i}=\sqrt{\frac{\sqrt{\alpha_{i}^{2}+\beta_{i}^{2}}-\alpha_{i}}{2}}, \delta_{i}=\sqrt{\frac{\sqrt{\alpha_{i}^{2}+\beta_{i}^{2}}+\alpha_{i}}{2}}, \\
& \bar{\alpha}_{i}=4 \sqrt{\alpha_{i}^{2}+\beta_{i}^{2}}+8 \alpha_{i}, \quad \bar{\beta}_{i}=4 \sqrt{\alpha_{i}^{2}+\beta_{i}^{2}}-8 \alpha_{i} .
\end{aligned}
$$

Similarly, by an inspection, we can see that

$$
-\left[\begin{array}{c}
\Phi_{i} \\
\frac{1}{1 !} \partial_{\alpha_{i}} \Phi_{i} \\
\vdots \\
\frac{1}{\left(l_{i}-1\right) !} \partial_{\alpha_{i}}^{l_{i}-1} \Phi_{i}
\end{array}\right]_{x x}=\left[\begin{array}{cccc}
A_{i} & & & 0 \\
I_{2} & A_{i} & & \\
& \ddots & \ddots & \\
0 & & I_{2} & A_{i}
\end{array}\right]_{l_{i} \times l_{i}}\left[\begin{array}{c}
\Phi_{i} \\
\frac{1}{1 !} \partial_{\alpha_{i}} \Phi_{i} \\
\vdots \\
\frac{1}{\left(l_{i}-1\right) !} \partial_{\alpha_{i}}^{l_{i}-1} \Phi_{i}
\end{array}\right],
$$

and

$$
\left(\frac{1}{j !} \partial_{\alpha_{i}}^{j} \Phi_{i}\right)_{t}=-4\left(\frac{1}{j !} \partial_{\alpha_{i}}^{j} \Phi_{i}\right)_{x x x}, 0 \leq j \leq l_{i}-1,
$$

where $\partial_{\alpha_{i}}$ denotes the derivative with respect to $\alpha_{i}$. Taking the derivative with respect to $\beta_{i}$, we can have

$$
-\left[\begin{array}{c}
\Phi_{i} \\
\frac{1}{1 !} \partial_{\beta_{i}} \Phi_{i} \\
\vdots \\
\frac{1}{\left(l_{i}-1\right) !} \partial_{\beta_{i}}^{l_{i}-1} \Phi_{i}
\end{array}\right]_{x x}=\left[\begin{array}{cccc}
A_{i} & & & 0 \\
\Sigma_{2} & A_{i} & & \\
& \ddots & \ddots & \\
0 & & \Sigma_{2} & A_{i}
\end{array}\right]_{l_{i} \times l_{i}}\left[\begin{array}{c}
\Phi_{i} \\
\frac{1}{1 !} \partial_{\beta_{i}} \Phi_{i} \\
\vdots \\
\frac{1}{\left(l_{i}-1\right) !} \partial_{\beta_{i}}^{l_{i}-1} \Phi_{i}
\end{array}\right]
$$

and

$$
\left(\frac{1}{j !} \partial_{\beta_{i}}^{j} \Phi_{i}\right)_{t}=-4\left(\frac{1}{j !} \partial_{\beta_{i}}^{j} \Phi_{i}\right)_{x x x}, 0 \leq j \leq l_{i}-1,
$$

where

$$
\Sigma_{2}=\left[\begin{array}{cc}
0 & -1 \\
1 & 0
\end{array}\right] .
$$

Therefore, we obtain two Wronskian solutions to the KdV equation (1.1):

$$
u=-2 \partial_{x}^{2} \ln W\left(\Phi_{i}^{T}, \frac{1}{1 !} \partial_{\alpha_{i}} \Phi_{i}^{T}, \cdots, \frac{1}{\left(l_{i}-1\right) !} \partial_{\alpha_{i}}^{l_{i}-1} \Phi_{i}^{T}\right),
$$

and

$$
u=-2 \partial_{x}^{2} \ln W\left(\Phi_{i}^{T}, \frac{1}{1 !} \partial_{\beta_{i}} \Phi_{i}^{T}, \cdots, \frac{1}{\left(l_{i}-1\right) !} \partial_{\beta_{i}}^{l_{i}-1} \Phi_{i}^{T}\right),
$$

which correspond to the same Jordan block of the second type but are different. Such a general Wronskian solution is

$$
u=-2 \partial_{x}^{2} \ln W\left(\Phi_{1}^{T}, \cdots, \frac{1}{\left(l_{1}-1\right) !} \partial_{\zeta_{1}}^{l_{1}-1} \Phi_{1}^{T} ; \cdots ; \Phi_{n}^{T}, \cdots, \frac{1}{\left(l_{n}-1\right) !} \partial_{\zeta_{n}}^{l_{n}-1} \Phi_{n}^{T}\right),
$$

where $\partial_{\zeta_{i}}$ can be either of $\partial_{\alpha_{i}}$ and $\partial_{\beta_{i}}$. This solution is called an $n$-complexiton solution of order $\left(l_{1}-1, l_{2}-1, \cdots, l_{n}-1\right)$, to reflect the orders of derivatives of eigenfunctions with respect to eigenvalues. If $l_{i}=1,1 \leq i \leq n$, we simply say that it is an $n$-complexiton. 
In particular, one-complexiton is $\underline{\mathrm{M}}]$

$$
\begin{aligned}
u= & -2 \partial_{x}^{2} \ln W\left(\phi_{11}, \phi_{12}\right) \\
= & \frac{-4 \beta_{1}^{2}\left[1+\cos \left(2 \delta_{1}\left(x-\bar{\beta}_{1} t\right)+2 \kappa_{1}\right) \cosh \left(2 \Delta_{1}\left(x+\bar{\alpha}_{1} t\right)+2 \gamma_{1}\right)\right]}{\left[\Delta_{1} \sin \left(2 \delta_{1}\left(x-\bar{\beta}_{1} t\right)+2 \kappa_{1}\right)+\delta_{1} \sinh \left(2 \Delta_{1}\left(x+\bar{\alpha}_{1} t\right)+2 \gamma_{1}\right)\right]^{2}} \\
& +\frac{4 \alpha_{1} \beta_{1} \sin \left(2 \delta_{1}\left(x-\bar{\beta}_{1} t\right)+2 \kappa_{1}\right) \sinh \left(2 \Delta_{1}\left(x+\bar{\alpha}_{1} t\right)+2 \gamma_{1}\right)}{\left[\Delta_{1} \sin \left(2 \delta_{1}\left(x-\bar{\beta}_{1} t\right)+2 \kappa_{1}\right)+\delta_{1} \sinh \left(2 \Delta_{1}\left(x+\bar{\alpha}_{1} t\right)+2 \gamma_{1}\right)\right]^{2}},
\end{aligned}
$$

where $\alpha_{1}, \beta_{1}>0, \kappa_{1}=\kappa_{11}=\kappa_{12}$, and $\gamma_{1}=\gamma_{11}=\gamma_{12}$ are arbitrary real constants, and $\Delta_{1}, \delta_{1}, \bar{\alpha}_{1}$, and $\bar{\beta}_{1}$ are given by

$$
\begin{aligned}
& \Delta_{1}=\sqrt{\frac{\sqrt{\alpha_{1}^{2}+\beta_{1}^{2}}-\alpha_{1}}{2}}, \delta_{1}=\sqrt{\frac{\sqrt{\alpha_{1}^{2}+\beta_{1}^{2}}+\alpha_{1}}{2}}, \\
& \bar{\alpha}_{1}=4 \sqrt{\alpha_{1}^{2}+\beta_{1}^{2}}+8 \alpha_{1}, \bar{\beta}_{1}=4 \sqrt{\alpha_{1}^{2}+\beta_{1}^{2}}-8 \alpha_{1} .
\end{aligned}
$$

The subcase of $\alpha_{1}=0$ leads to the following solution:

$$
u=\frac{8 \beta_{1}+8 \beta_{1} \cos \left(\sqrt{2 \beta_{1}} x-4 \beta_{1} \sqrt{2 \beta_{1}} t+2 \kappa_{1}\right) \cosh \left(\sqrt{2 \beta_{1}} x+4 \beta_{1} \sqrt{2 \beta_{1}} t+2 \gamma_{1}\right)}{\left[\sin \left(\sqrt{2 \beta_{1}} x-4 \beta_{1} \sqrt{2 \beta_{1}} t+2 \kappa_{1}\right)+\sinh \left(\sqrt{2 \beta_{1}} x+4 \beta_{1} \sqrt{2 \beta_{1}} t+2 \gamma_{1}\right)\right]^{2}} .
$$

This solution is associated with purely imaginary eigenvalues of the Schrödinger spectral problem with zero potential. In addition, if we fix

$$
\kappa_{1}=\frac{\pi}{4}, \gamma_{1}=\frac{1}{2} \ln \left(\frac{b}{a}\right), \Delta_{1}=-a, \delta_{1}=b,
$$

where $a$ and $b$ are arbitrary real constants, then our one-complexiton solution (4.28) boils down to the breather-like or spike-like solution presented in [J]:

$$
u=\frac{8\left\{\left(a^{2}-b^{2}\right)(b / a) \cos \nu \sinh (\eta+p)+2 b^{2}[1+\sin \nu \cosh (\eta+p)]\right\}}{[\cos \nu-(b / a) \sinh (\eta+p)]^{2}},
$$

where $\nu=-2 b x+8\left(3 a^{2} b-b^{3}\right) t, \eta=-2 a x+8\left(a^{3}-3 a b^{2}\right) t$ and $p=\ln (b / a)$. Figure 3 depicts some singularities of the single complexiton solution with $\kappa=\gamma=0$.
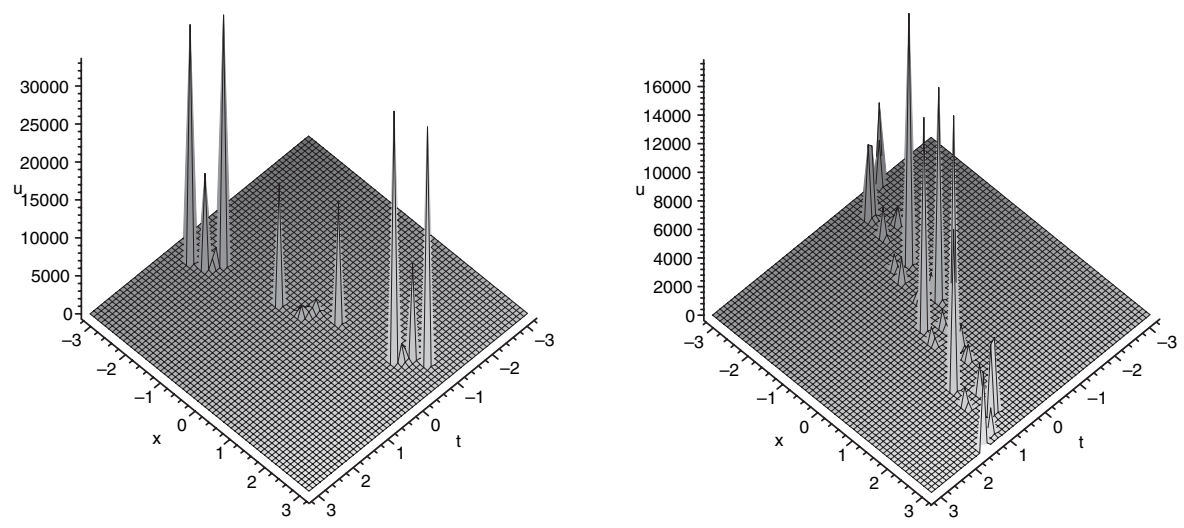

Figure 3. 1-complexiton - $\alpha_{1}=0, \beta_{1}=1$ (left) 1-complexiton - $\alpha_{1}=1, \beta_{1}=1$ (right) 
4.4. Interaction solutions. We are now presenting examples of Wronskian interaction solutions among different kinds of Wronskian solutions to the KdV equation.

Let us assume that there are two sets of eigenfunctions

$$
\phi_{1}(\lambda), \phi_{2}(\lambda), \cdots, \phi_{k}(\lambda) ; \psi_{1}(\mu), \psi_{2}(\mu), \cdots, \psi_{l}(\mu)
$$

associated with two different eigenvalues $\lambda$ and $\mu$, respectively. A Wronskian solution

$$
u=-2 \partial_{x}^{2} \ln W\left(\phi_{1}(\lambda), \cdots, \phi_{k}(\lambda) ; \psi_{1}(\mu), \cdots, \psi_{l}(\mu)\right)
$$

is said to be a Wronskian interaction solution between two solutions determined by the two sets of eigenfunctions in (4.29). Of course, we can have more general Wronskian interaction solutions among three or more kinds of solutions such as rational solutions, positons, solitons, negatons, breathers and complexitons. Roughly speaking, it increases the complexities of rational solutions, positons, negatons and complexitons, respectively, to add zero, positive, negative or complex eigenvalues to the spectrum of the coefficient matrix.

In what follows, we would like to show a few special Wronskian interaction solutions. Let us first choose different sets of eigenfunctions:

$$
\begin{aligned}
& \phi_{\text {rational }}=c x+d, c, d=\text { consts.; } \\
& \phi_{\text {soliton }}=\cosh \left(\eta x-4 \eta^{3} t+\gamma\right), \eta, \gamma=\text { consts.; } \\
& \phi_{\text {positon }}=\cos \left(\eta x+4 \eta^{3} t+\gamma\right), \eta, \gamma=\text { consts.; } \\
& \phi_{\text {complexiton }, 1}=\cos \left(\frac{\sqrt{2}}{2}(x-4 t)+\kappa\right) \cosh \left(\frac{\sqrt{2}}{2}(x+4 t)+\gamma\right), \kappa, \gamma=\text { consts. } \\
& \phi_{\text {complexiton }, 2}=\sin \left(\frac{\sqrt{2}}{2}(x-4 t)+\kappa\right) \sinh \left(\frac{\sqrt{2}}{2}(x+4 t)+\gamma\right), \kappa, \gamma=\text { consts. }
\end{aligned}
$$

Three Wronskian interaction determinants between any two of a rational solution, a single soliton and a single positon are

$$
\begin{gathered}
W\left(\phi_{\text {rational }}, \phi_{\text {soliton }}\right)=\eta(c x+d) \sinh \left(\eta x-4 \eta^{3} t+\gamma\right)-c \cosh \left(\eta x-4 \eta^{3} t+\gamma\right), \\
\begin{aligned}
W\left(\phi_{\text {rational }}, \phi_{\text {positon }}\right)= & -\eta(c x+d) \sin \left(\eta x+4 \eta^{3} t+\gamma\right)-c \cos \left(\eta x+4 \eta^{3} t+\gamma\right), \\
W\left(\phi_{\text {soliton }}, \phi_{\text {positon }}\right)= & -\eta \cosh \left(\eta x-4 \eta^{3} t+\gamma\right) \sin \left(\eta x+4 \eta^{3} t+\gamma\right) \\
& -\eta \cos \left(\eta x+4 \eta^{3} t+\gamma\right) \sinh \left(\eta x-4 \eta^{3} t+\gamma\right) .
\end{aligned}
\end{gathered}
$$

Further, the corresponding Wronskian interaction solutions are

$$
\begin{aligned}
& u_{r s}=-2 \partial_{x}^{2} \ln W\left(\phi_{\text {rational }}, \phi_{\text {soliton }}\right)=\frac{2 \eta^{2}\left[\eta^{2}(c x+d)^{2}+c^{2} \cosh ^{2} \xi_{-}\right]}{\left[\eta(c x+d) \sinh \xi_{-}-c \cosh \xi_{-}\right]^{2}}, \\
& u_{r p}=-2 \partial_{x}^{2} \ln W\left(\phi_{\text {rational }}, \phi_{\text {positon }}\right)=\frac{2 \eta^{2}\left[\eta^{2}(c x+d)^{2}-c^{2} \cos ^{2} \xi_{+}\right]}{\left[\eta(c x+d) \sin \xi_{+}+c \cos \xi_{+}\right]^{2}}, \\
& u_{s p}=-2 \partial_{x}^{2} \ln W\left(\phi_{\text {soliton }}, \phi_{\text {positon }}\right)=\frac{4 \eta^{2}\left(\cosh ^{2} \xi_{-}+\cos ^{2} \xi_{+}\right)}{\left(\cosh \xi_{-} \sin \xi_{+}+\cos \xi_{+} \sinh \xi_{-}\right)^{2}},
\end{aligned}
$$

where

$$
\xi_{ \pm}=\eta x \pm 4 \eta^{3} t+\gamma
$$

Some singularities of these solutions are shown in Figures 4 and 5 

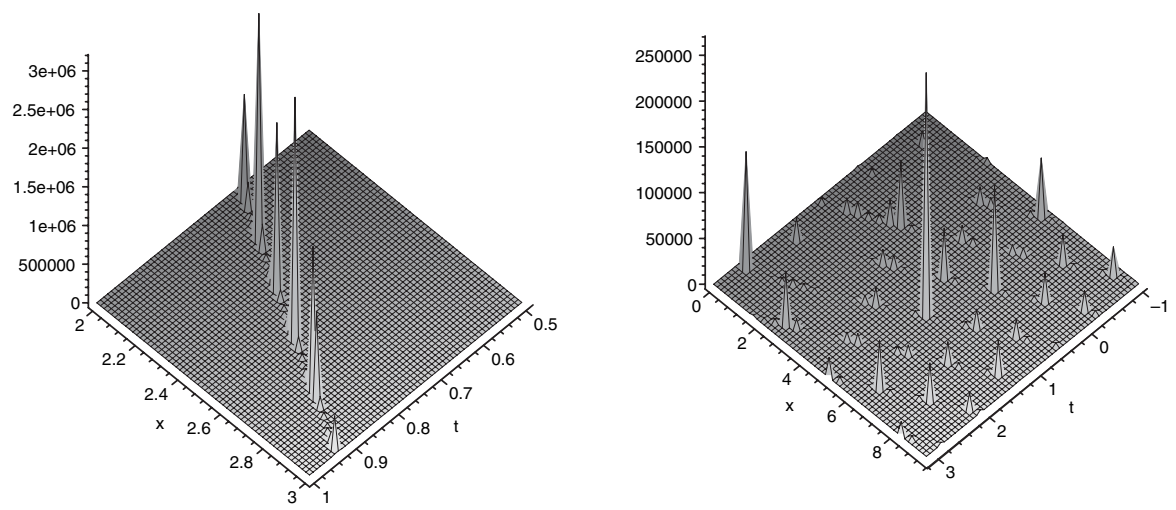

FiguRE 4. $u_{r s}-\eta=\gamma=c=d=1$ (left) $\quad u_{r p}-\eta=\gamma=c=$ $d=1$ (right)
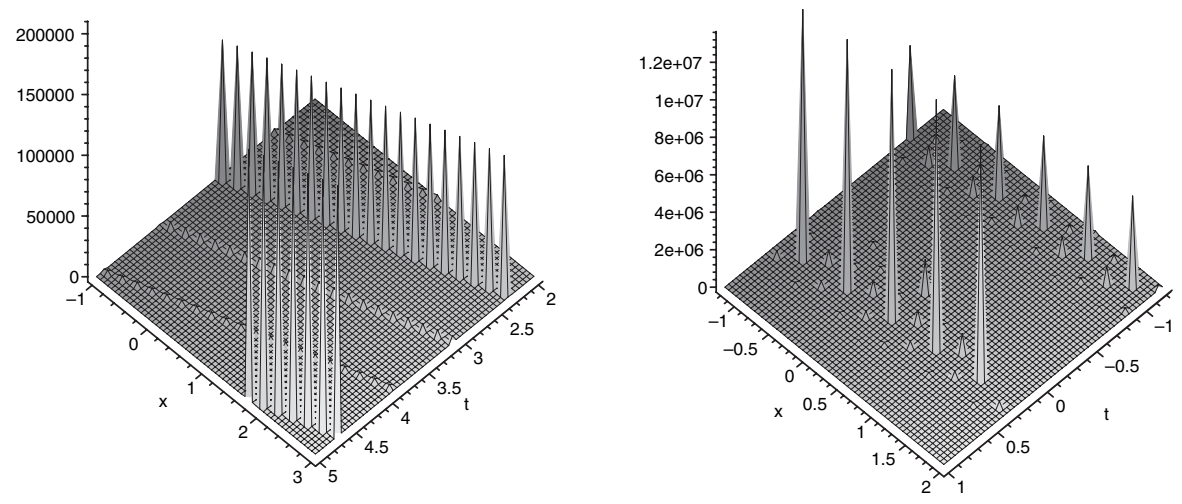

FiguRE 5. $u_{s p}-\eta=\gamma=c=d=1$ (left) $\quad u_{s p}-\eta=2, \gamma=c=$ $d=1$ (right)

The following are two Wronskian interaction determinants and solutions involving three eigenfunctions. The first Wronskian determinant is

$$
\begin{aligned}
& W\left(\phi_{\text {rational }}, \phi_{\text {soliton }}, \phi_{\text {positon }}\right) \\
= & -\eta^{2}\left[(c x+d) \eta\left(\cos \xi_{+} \sinh \xi_{-}-\sin \xi_{+} \cosh \xi_{-}\right)-2 c \cos \xi_{+} \cosh \xi_{-}\right],
\end{aligned}
$$

so that its corresponding Wronskian solution is

$$
\begin{aligned}
u_{r s p}= & -2 \partial_{x}^{2} \ln W\left(\phi_{\text {rational }}, \phi_{\text {soliton }}, \phi_{\text {positon }}\right) \\
= & \frac{4(c x+d) \eta^{3}\left(\sin \xi_{+} \cosh \xi_{-}+\cos \xi_{+} \sinh \xi_{-}\right)}{(c x+d) \eta\left(\cos \xi_{+} \sinh \xi_{-}-\sin \xi_{+} \cosh \xi_{-}\right)-2 c \cos \xi_{+} \cosh \xi_{-}} \\
& +\frac{2 \eta^{2}\left[c \eta \cos \xi_{+} \sinh \xi_{-}+2(c x+d) \eta \sin \xi_{+} \sinh \xi_{-}-c \eta \sin \xi_{+} \cosh \xi_{-}\right]^{2}}{\left[(c x+d) \eta\left(\cos \xi_{+} \sinh \xi_{-}-\sin \xi_{+} \cosh \xi_{-}\right)-2 c \cos \xi_{+} \cosh \xi_{-}\right]^{2}},
\end{aligned}
$$

where

$$
\xi_{ \pm}=\eta x \pm 4 \eta^{3} t+\gamma
$$


The second Wronskian determinant and its corresponding Wronskian solution are

$$
\begin{aligned}
& W\left(x, \phi_{\text {complexiton }, 1}, \phi_{\text {complexiton }, 2}\right) \\
= & -\frac{\sqrt{2}}{4} x \sin 2 \xi+\frac{\sqrt{2}}{4} x \sinh 2 \zeta-\cosh ^{2} \zeta+\sin ^{2} \xi,
\end{aligned}
$$

and

$$
\begin{aligned}
u_{r c}= & -2 \partial_{x}^{2} \ln W\left(x, \phi_{\text {complexiton }, 1}, \phi_{\text {complexiton }, 2}\right) \\
= & {\left[\cosh ^{4} \zeta-\cos ^{4} \xi-4 x^{2} \cos ^{2} \xi \cosh ^{2} \zeta+\left(2 x^{2}-1\right) \cosh ^{2} \zeta\right.} \\
& +\left(2 x^{2}+1\right) \cos ^{2} \xi+4 \sqrt{2} x \cosh ^{2} \zeta \sin 2 \xi+4 \sqrt{2} x \cos ^{2} \xi \sinh 2 \zeta \\
& \left.-\sqrt{2} x \sinh 2 \zeta-\sqrt{2} x \sin 2 \xi-\frac{1}{2} \sin 2 \xi \sinh 2 \zeta\right] /\left(-\frac{\sqrt{2}}{4} x \sin 2 \xi\right. \\
& \left.+\frac{\sqrt{2}}{4} x \sinh 2 \zeta-\cosh ^{2} \zeta+\sin ^{2} \xi\right)^{2},
\end{aligned}
$$

where

$$
\xi=\frac{1}{2} \sqrt{2} x-2 \sqrt{2} t+\kappa, \zeta=\frac{1}{2} \sqrt{2} x+2 \sqrt{2} t+\gamma .
$$

Figure 6 depicts some singularities of these two solutions.
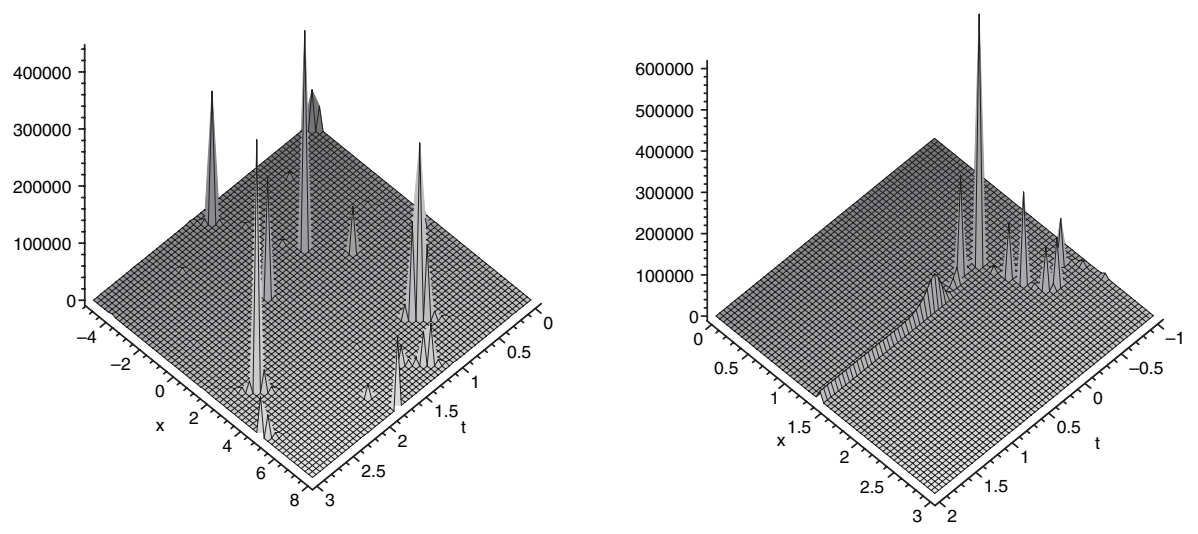

Figure 6. $u_{r s p}-\eta=\gamma=c=d=1$ (left) $\quad u_{r c}-\kappa=\gamma=1$ (right)

\section{Concluding Remarks}

A broad set of sufficient conditions consisting of coupled systems of linear partial differential equations has been presented, which guarantees that the Wronskian determinant solves the Korteweg-de Vries equation in the bilinear form. A systematical analysis has been made for solving the resultant coupled systems of linear partial differential equations, and solution formulas for their representative systems have been explicitly presented. The key technique is to apply the variation of parameters in solving the involved non-homogeneous partial differential equations of second-order and third-order. The whole analysis also offers an approach to solve 
the coupled system of partial differential equations:

$$
-\phi_{i, x x}=\sum_{i=1}^{N} \lambda_{i j} \phi_{j}, \phi_{i, t}=-4 \phi_{i, x x x}, 1 \leq i \leq N,
$$

where $\lambda_{i j}$ are arbitrary real constants.

Moreover, for each type of Jordan blocks of the coefficient matrix $\Lambda=\left(\lambda_{i j}\right)$, special sets of eigenfunctions have been constructed and used to generate rational solutions, solitons, positons, negatons, breathers, complexitons and their interaction solutions to the Korteweg-de Vries equation. Of course, the obtained solution formulas of the representative systems allow us to construct more general Wronskian solutions than rational solutions, positons, negatons, complexitons and their interaction solutions presented in this paper. The resultant solution structures also show us the rich diversity that the KdV equation carries. We believe that any $(2+1)$-dimensional counterpart of the KdV equation will have much more general solution structures. This is because higher dimensional equations have bigger spaces of initial data to choose.

However, any new explicit exact solutions, even Wronskian solutions, to the KdV equation will still be very interesting. Two open questions follow:

(1) What about the following case?

$$
\begin{aligned}
& -\phi_{i, x x}=\sum_{j=1}^{N} \mu_{i j} \phi_{j, x}+\sum_{j=1}^{N} \lambda_{i j} \phi_{j}, 1 \leq i \leq N, \\
& \phi_{i, t}=\sum_{j=1}^{N} \zeta_{i j} \phi_{j, x x x}+\sum_{j=1}^{N} \xi_{i j} \phi_{j}, 1 \leq i \leq N,
\end{aligned}
$$

where all coefficients are real constants. Are there any conditions, rather than the ones established in this paper, which will guarantee the Wronskian solutions for the KdV equation?

(2) Are there any similar conditions to guarantee the double Wronskian solutions for the KdV equation?

On the other hand, it deserves more investigation whether there exist Wronskian solutions for the generalized KdV equations (see, say, [B, $, \mathrm{Y}, \mathrm{SY}]$ for examples) and what differential conditions on Wronskian solutions one can have if there exist Wronskian solutions.

\section{ACKNOWLEDGMENTS}

The authors would like to thank C. R. Gilson, K. Manuro and M. Pavlov for stimulating discussions. They are also grateful to the referee for helpful suggestions and valuable comments. The work was supported in part by the University of South Florida Internal Awards Program under Grant No. 1249-936RO.

\section{REFERENCES}

[AKNS] M. J. Ablowitz, D. J. Kaup, A. C. Newell and H. Segur, The inverse scattering transform-Fourier analysis for nonlinear problems, Studies in Appl. Math. 53 (1974), 249-315. MR0450815 (56:9108)

[ASa] M. J. Ablowitz and J. Satsuma, Solitons and rational solutions of nonlinear evolution equations, J. Math. Phys. 19 (1978), 2180-2186. MR0507515 (80b:35121) 
[ASe] M. J. Ablowitz and H. Segur, Solitons and the Inverse Scattering Transform, SIAM, Philadelphia, 1981. MR0642018 (84a:35251)

[AM] M. Adler and J. Moser, On a class of polynomials connected with the Korteweg-de Vries equation, Commun. Math. Phys. 61 (1978), 1-30. MR0501106 (58:18554)

[APP] V. A. Arkad'ev, A. K. Pogrebkov and M. K. Polivanov, Singular solutions of the KdV equation and the method of the inverse problem, Zap. Nauchn. Sem. Leningrad. Otdel. Mat. Inst. Steklov. (LOMI) 133 (1984), 17-37. MR0742146 (86c:35127)

[AP] D. K. Arrowsmith and C. M. Place, Dynamical Systems, Chapman \& Hall, London, 1992. MR.1195127 (93j:58040)

[BR] D. J. Benney and D. J. Roskes, Wave instabilities, Studies in Appl. Math. 48 (1969), $377-385$

[BS] A. C. Bryan and A. E. G. Stuart, Representations of the multisoliton solutions of the Korteweg-de Vries equation, Nonlinear Anal. 22 (1994), 561-566. MR1266543 (94m:35260)

[BC] R. K. Bullough and P. J. Caudrey (eds.), Solitons, Springer-Verlag, Berlin, 1980. MF0625877 (82m:35001)

[B] N. J. Burroughs, A loop algebra co-adjoint orbit construction of the generalized KdV hierarchies, Nonlinearity 6 (1993), 583-616. MR1231775 (94j:58078)

[DS] A. Davey and K. Stewarton, On three-dimensional packets of surface waves, Proc. R. Soc. A 338 (1974), 101-110. MR0349126 (50:1620)

[DJ] P. G. Drazin and R. S. Johnson, Solitons: an Introduction, Cambridge University Press, Cambridge, 1989. MR0985322 (90j:35166)

[FN] N. C. Freeman and J. J. C. Nimmo, Soliton solutions of the Korteweg-de Vries and Kadomtsev-Petviashvili equations: the Wronskian technique, Phys. Lett. A 95 (1983), 1-3. MR0700477 (85j:35168)

$[\mathrm{H}] \quad$ R. Hirota, Exact solution of the Korteweg de Vries equation for multiple collisions of solitons, Phys. Rev. Lett. 27 (1971), 1192-1194.

[HS] M. W. Hirsch and S. Smale, Differential Equations, Dynamical Systems, and Linear Algebra, Academic Press, San Diego, California, 1974. MR.0486784 (58:6484)

[J] M. Jaworski, Breather-like solutions to the Korteweg-de Vries equation, Phys. Lett. A 104 (1984), 245-247. MR0758224 (85h:35198)

[KP] B. B. Kadomtsev and V. I. Petviashvili, On the stability of solitary waves in weakly dispersing media, Sov. Phys. Dokl. 15 (1970), 539-541.

[K] M. Kovalyov, Basic motions of the Korteweg-de Vries equation, Nonlinear Anal. 31 (1998), 599-619. MR 1487849|(99f:35177)

[L] P. D. Lax, Integrals of nonlinear equations of evolution and solitary waves, Comm. Pure Appl. Math. 21 (1968), 467-490. MR0235310 (38:3620)

[M] W. X. Ma, Complexiton solutions to the Korteweg-de Vries equation, Phys. Lett. A 301 (2002), 35-44. MR1927047|(2003h:35237)

[Mag] F. Magri, A simple model of the integrable Hamiltonian equation, J. Math. Phys. 19 (1978), 1156-1162. MF0488516 (80a:35112)

[Mat] V. B. Matveev, Generalized Wronskian formula for solutions of the KdV equations: first applications; Positon-positon and soliton-positon collisions: KdV case, Phys. Lett. A 166 (1992), 205-208; 209-212. MR1170966 (93g:35125a); MR1170967 (93c:35141)

[MS] V. B Matveev and M. A. Salle, Darboux Transformations and Solitons, SpringerVerlag, Berlin, 1991. MR:1146435 (93d:35136)

[MGK] R. M. Miura, C. S. Gardner and M. D. Kruskal, Korteweg-de Vries equation and generalizations II: Existence of conservation laws and constants of motion, J. Math. Phys. 9 (1968), 1204-1209. MR0252826 (40:6042b)

[RSK] C. Rasinariu, U. Sukhatme and A. Khare, Negaton and positon solutions of the KdV and $m K d V$ hierarchy, J. Phys. A: Math. Gen. 29 (1996), 1803-1823. MR1395807 (97c:35182)

[S] J. Satsuma, A Wronskian representation of $N$-soliton solutions of nonlinear evolution equations, J. Phys. Soc. Jpn. 46 (1979) 359-360.

[SW] G. Segal and G. Wilson, Loop groups and equations of KdV type, Inst. Hautes Études Sci. Publ. Math. No. 61 (1985), 5-65. MR0783348 (87b:58039) 
[SY] G. R. Sell and Y. You, Dynamics of Evolutionary Equations, Springer-Verlag, NewYork, 2002. MR,1873467 (2003f:37001b)

[SHR] S. Sirianunpiboon, S. D. Howard and S. K. Roy, A note on the Wronskian form of solutions of the KdV equation, Phys. Lett. A 134 (1988), 31-33. MR0972621 (89k:35230)

[Y] Y. You, Global dynamics of dissipative generalized Korteweg-de Vries equations, Chin. Ann. of Math. 17B (1996), 389-402. MR.1441652 (97k:35230)

Department of Mathematics, University of South Florida, Tampa, Florida 33620-5700

E-mail address: mawx@math.usf.edu

Department of Mathematics, University of South Florida, Tampa, Florida 33620-5700

E-mail address: you@math.usf.edu 